TRANSACTIONS OF THE

AMERICAN MATHEMATICAL SOCIETY

Volume 359, Number 4, April 2007, Pages 1403-1432

S 0002-9947(06)03918-3

Article electronically published on November 17, 2006

\title{
BOCHNER-RIESZ MEANS WITH RESPECT TO A ROUGH DISTANCE FUNCTION
}

\author{
PAUL TAYLOR
}

ABSTRACT. The generalized Bochner-Riesz operator $S^{R, \lambda}$ may be defined as

$$
S^{R, \lambda} f(x)=\mathcal{F}^{-1}\left[\left(1-\frac{\rho}{R}\right)_{+}^{\lambda} \widehat{f}\right](x)
$$

where $\rho$ is an appropriate distance function and $\mathcal{F}^{-1}$ is the inverse Fourier transform. The behavior of $S^{R, \lambda}$ on $L^{p}\left(\mathbf{R}^{d} \times \mathbf{R}\right)$ is described for $\rho\left(\xi^{\prime}, \xi_{d+1}\right)=$ $\max \left\{\left|\xi^{\prime}\right|,\left|\xi_{d+1}\right|\right\}$, a rough distance function. We conjecture that this operator is bounded on $\mathbf{R}^{d} \times \mathbf{R}$ when $\lambda>\max \left\{d\left(\frac{1}{2}-\frac{1}{p}\right)-\frac{1}{2}, 0\right\}$ and $p<2+\frac{6}{d-3}$, and unbounded when $p \geq 2+\frac{6}{d-3}$. This conjecture is verified for large ranges of $p$.

\section{INTRODUCTION}

Let $\mathcal{S}$ denote the space of Schwartz functions (smooth, rapidly decaying at $\infty$ ) on $\mathbf{R}^{d}$. For $f \in \mathcal{S}$, the Fourier transform $\widehat{f}$ is defined as follows:

$$
\widehat{f}(\xi)=\int_{\mathbf{R}^{d}} e^{-i x \cdot \xi} f(x) d x .
$$

We also have the inverse Fourier transform,

$$
\mathcal{F}^{-1}[g](x)=\frac{1}{(2 \pi)^{d}} \int_{\mathbf{R}^{d}} e^{i x \cdot \xi} g(\xi) d \xi .
$$

For $f \in \mathcal{S}$, we have $\mathcal{F}^{-1}[\widehat{f}]=f$. One can extend the definition of $\widehat{f}$ to $f \in \mathcal{S}^{\prime}$ $\left(\mathcal{S}^{\prime}\right.$ denotes the dual of $\mathcal{S}$, the space of tempered distributions). However, when one extends the Fourier transform, it is not an integrable function in general. One hopes to make sense of the inverse Fourier transform integral, $\mathcal{F}^{-1}[\widehat{f}]$ for $f \in L^{p}$, as a limit. Generalized Bochner-Riesz means attempt to address this question.

We define distance functions to be $\rho$ which are continuous on $\mathbf{R}^{d}$ and satisfy

$$
\begin{aligned}
\rho(t x) & =t \rho(x), \quad t>0, \\
\rho(x) & >0 \quad \text { if } x \neq 0 .
\end{aligned}
$$

We define the generalized Bochner-Riesz operator $S^{R, \lambda}$ as follows:

$$
S^{R, \lambda} f(x)=\mathcal{F}^{-1}\left[\left(1-\frac{\rho}{R}\right)_{+}^{\lambda} \widehat{f}\right](x) .
$$

Received by the editors July 26, 2004 and, in revised form, November 16, 2004.

2000 Mathematics Subject Classification. Primary 42B15; Secondary 42B25.

Key words and phrases. Fourier analysis, multipliers, Bochner-Riesz means, cone multiplier.

The author thanks Andreas Seeger for his guidance.

(C)2006 American Mathematical Society Reverts to public domain 28 years from publication 
Here $(g(\xi))_{+}=\max \{g(\xi), 0\}$ is the positive part. Note that as $R \rightarrow \infty, S^{R, \lambda} f \rightarrow f$ for $f \in \mathcal{S}$. Also note that $S^{R, \lambda} f \rightarrow f$ for $f \in L^{2}$ by Plancherel's theorem. On general principals, the question of convergence on other $L^{p}$ spaces is equivalent to the question of boundedness of the operators $S^{R, \lambda}$. By scaling, we may also assume that $R=1$. From now on we will focus on the boundedness of $S^{\lambda}=S^{1, \lambda}$ on $L^{p}$.

The generalized Bochner-Riesz operator has been studied extensively in the case where $\rho(\xi)=|\xi|$. In this case, the operator represents "standard" Bochner-Riesz means (referred to later as spherical means, as the set $|\xi|=1$ is a sphere). In 1971, Fefferman [6] showed that for spherical means to be bounded on $L^{p}$, it is necessary to have $\lambda>\lambda^{*}(p)=\max \left\{d\left|\frac{1}{2}-\frac{1}{p}\right|-\frac{1}{2}, 0\right\}$. The Bochner-Riesz conjecture states that this is both necessary and sufficient.

In 1972, Carleson and Sjölin verified the conjecture in $\mathbf{R}^{2}[3]$. In 1973, Fefferman showed a connection between the restriction conjecture for the sphere and the Bochner-Riesz conjecture [7. Recent progress on the restriction conjecture has used a bilinear approach (as in 20]) and recently Lee adapted Fefferman's argument to apply the bilinear results directly to spherical means [10. This approach applied to the recent bilinear result of Tao [19] proves the Bochner-Riesz conjecture for $p>2+\frac{4}{d}$ for $d \geq 3$, the best current range of $p$.

While the case of $\rho(\xi)=|\xi|$ provides an interesting test case, it is much simpler than the question of general $\rho$. If one wishes to study $S^{\lambda}$ with a $\rho$ which is not smooth, a good example to consider is $\rho(\xi)=\max \left\{\left|\xi^{\prime}\right|,\left|\xi_{d+1}\right|\right\}$ where $\xi=$ $\left(\xi^{\prime}, \xi_{d+1}\right) \in \mathbf{R}^{d} \times \mathbf{R}$. For this $\rho$, we denote the multiplier by $m_{\lambda}$,

$$
m_{\lambda}(\xi)=\left(1-\max \left\{\left|\xi^{\prime}\right|,\left|\xi_{d+1}\right|\right\}\right)_{+}^{\lambda} .
$$

The support of $m_{\lambda}$ is a cylinder. For $\xi$ near $\left|\xi^{\prime}\right|=1, m_{\lambda}=\left(1-\left|\xi^{\prime}\right|\right)_{+}^{\lambda}$ which is the multiplier for spherical means in $\mathbf{R}^{d}$. Note that this relationship is why we define this new $\rho$ for $\xi \in \mathbf{R}^{d} \times \mathbf{R}$ (as opposed to $\mathbf{R}^{d-1} \times \mathbf{R}$ ). This way the same critical index $\lambda^{*}(p)$ will apply for spherical means and the cylinder multiplier, as well as the cone multiplier (defined below, also on $\mathbf{R}^{d} \times \mathbf{R}$ ). For $\xi$ near $\left|\xi_{d+1}\right|=1$, $m_{\lambda}=\left(1-\left|\xi_{d+1}\right|\right)_{+}^{\lambda}$ which is the multiplier for spherical means in $\mathbf{R}^{1}$. Spherical means in $\mathbf{R}^{1}$ are easily shown to be bounded for all $p \geq 1$ when $\lambda>0$, which is weaker than the $\lambda>\lambda^{*}(p)$ condition on spherical means in $\mathbf{R}^{d}$. Given these observations, one might expect that this cylindrical operator would be bounded for the same range of $\lambda$ as spherical means in $\mathbf{R}^{d}$. Interestingly, there is a significant difference.

This problem was previously studied by Luers [11] in 1988. He was able to obtain several results. First, when $d=2$, the inverse Fourier transform of $m_{\lambda}$ is in $L^{1}$ for $\lambda>\frac{1}{2}$. It follows that $S^{\lambda}$ is bounded for all $p \geq 1$ when $d=2$ and $\lambda>\frac{1}{2}$. Second, when $d \geq 3$ and $\lambda^{*}(p) \geq 1$, then $S^{\lambda}$ is unbounded for all $\lambda$. This is curious, since with spherical means there is always some large $\lambda$ for which the operator is bounded. Third, $S^{\lambda}$ is bounded for the relatively small range where $\lambda>1-\frac{1}{d+1}$ and $\left|\frac{1}{p}-\frac{1}{2}\right|<\frac{1}{d+1}$. Finally, he showed the kernel $\mathcal{F}^{-1}\left[m_{\lambda}\right]$ is in $L^{p}$ when $\lambda>\lambda^{*}(p)$ and $\lambda^{*}(p)<1$. We conjecture that the operator $S^{\lambda}$ is bounded when $\lambda>\lambda^{*}(p)$ and $\lambda^{*}(p)<1$.

The method used by Luers was to make explicit computations of the kernel and then estimate it as carefully as possible. Here, we will apply more modern methods, decomposing the multiplier on the Fourier transform side. We explain the curious necessary condition $\lambda^{*}(p)<1$ which limits the range of $p$. We also 


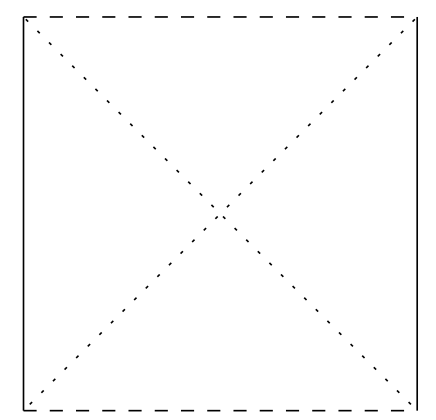

Figure 1. Regions of interest: Here the solid line is where $\left|\xi^{\prime}\right|=1$, the dashed line is where $\left|\xi_{d+1}\right|$ and the dotted line is where $\left|\xi^{\prime}\right|=$ $\left|\xi_{d+1}\right|$.

greatly improve the range of $\lambda$ for which $S^{\lambda}$ is bounded while relating it to more understood operators such as spherical means.

Our multiplier $m_{\lambda}(\xi)$ is supported on a cylinder but it behaves very differently than another multiplier supported on a cylinder given by $\left(1-\left|\xi^{\prime}\right|\right)^{\lambda}\left(1-|\xi|_{d+1}\right)^{\lambda}$. This second multiplier represents a simple composition of two spherical means operators. The significant difference is that the multiplier $m_{\lambda}$ is not smooth where $\left|\xi^{\prime}\right|=$ $\left|\xi_{d+1}\right|$, a region with the shape of a light cone. So, we expect that near $\left|\xi^{\prime}\right|=1$ and $\left|\xi_{d+1}\right|=1$ our multiplier will have the same boundedness properties as the spherical means of the appropriate lower dimensions. However, we also note that near $\left|\xi^{\prime}\right|=\left|\xi_{d+1}\right|$, our multiplier will relate to the cone multiplier. There is also an additional complication which we must address. Upon close inspection we see that the non-smoothness where $\left|\xi^{\prime}\right|=\left|\xi_{d+1}\right|$ becomes more severe as we approach $\left|\xi^{\prime}\right|=\left|\xi_{d+1}\right|=1$. This will be discussed once we get into the proof.

The cone multiplier is a variation of generalized Bochner-Riesz means which has also been deeply investigated. Here we consider an operator similar to $S^{\lambda}$ with $\rho(\xi)=\frac{\left|\xi^{\prime}\right|}{\left|\xi_{d+1}\right|}$. However, we also introduce a cutoff in $\left|\xi_{d+1}\right|$ to avoid 0 and $\infty$. Let $h(\xi)=1-\frac{\left|\xi^{\prime}\right|}{\left|\xi_{d+1}\right|}$. We define the cone multiplier operator $C^{\lambda}$,

$$
C^{\lambda} f(x)=\mathcal{F}^{-1}\left[(h)_{+}^{\lambda} \kappa\left(1-\left|(\cdot)_{d+1}\right|\right) \hat{f}\right](x)
$$

where $\left(\xi^{\prime}, \xi_{d+1}\right) \in \mathbf{R}^{d} \times \mathbf{R}$ and $\kappa \in \mathcal{C}_{0}^{\infty}$ is supported in $\left(-\frac{1}{2}, \frac{1}{2}\right)$. The nature of the curvature of the cone (total curvature non-vanishing, but always with one vanishing principal curvature) makes it a significantly different problem than spherical means.

It is conjectured that $C^{\lambda}$ is bounded for all $\lambda>\lambda^{*}(p)$, the same as spherical means in $\mathbf{R}^{d}$. The necessity of this conditions follows from its necessity for spherical means in $\mathbf{R}^{d}$, via a theorem of de Leeuw (see 8 for a proof due to Jodeit). Techniques can to some extent be transferred between spherical means and the cone multiplier. However, progress on the cone multiplier has somewhat lagged behind that of spherical means (see [12, [2]).

A major breakthrough was made by Wolff in 2000 [21], when he showed the first sharp bounds on the cone multiplier, in any dimension, for any $p$ (he proved the sharp bound in $\mathbf{R}^{2} \times \mathbf{R}$ for large $p$ ). His approach used the geometry of how thin tubes intersect each other, combined with an induction on scales argument. For this 
approach, it turns out that the cone offers certain advantages over Bochner-Riesz and for once the cone was leading the way. This result was extended to higher dimensions by Łaba and Wolff [9].

\section{RESUlts}

Once our multiplier is decomposed appropriately, it will have qualitative similarities with the sphere multiplier and the cone multiplier, as well as cone multipliers truncated to different heights. However, it will not be exactly the same so we will have to refer to the method of proof for results pertaining to these types of multipliers.

The conjectured sharp $L^{p} \rightarrow L^{p}$ result for our cylindrical multiplier would be to have boundedness when $\lambda>\lambda^{*}(p)$ for $p$ such that $\lambda^{*}(p)<1$. The $\lambda^{*}(p)<1$ condition in the conjecture comes from the conical area of non-smoothness, where our multiplier has qualitative similarities to the multiplier $\left(1-\frac{\left|\xi^{\prime}\right|}{\left|\xi_{d+1}\right|}\right)^{1}$, which is the cone multiplier and with $\lambda=1$ (see Proposition 2.11 where we verify this condition, originally discovered by Luers 11). Therefore, the cylindrical multiplier should be unbounded on $L^{p} \rightarrow L^{p}$ for $p$ such that $\lambda^{*}(p) \geq 1$. For notation purposes we set $\lambda^{*}(\infty)=\frac{d}{2}-\frac{1}{2}$.

The results vary depending on dimension, since different sphere and cone technology exists in different dimensions. Our various results are as follows:

Theorem 1.1. The operator $S_{\lambda}$ is bounded from $L^{p}\left(\mathbf{R}^{2} \times \mathbf{R}\right) \rightarrow L^{p}\left(\mathbf{R}^{2} \times \mathbf{R}\right)$ for $\lambda>\lambda^{*}(p), 1 \leq p \leq \infty$.

Theorem 1.2. The operator $S_{\lambda}$ is bounded from $L^{p}\left(\mathbf{R}^{3} \times \mathbf{R}\right) \rightarrow L^{p}\left(\mathbf{R}^{3} \times \mathbf{R}\right)$ for $\lambda>\lambda^{*}(p), 2+\frac{4}{d}=\frac{10}{3}<p<\infty$.

Theorem 1.3. The operator $S_{\lambda}$ is bounded from $L^{p}\left(\mathbf{R}^{4} \times \mathbf{R}\right) \rightarrow L^{p}\left(\mathbf{R}^{4} \times \mathbf{R}\right)$ for $\lambda>\lambda^{*}(p), 2+\frac{4}{d}=3<p<2+\frac{6}{d-3}-\frac{6}{61}=\frac{482}{61}$.

Theorem 1.4. If $d \geq 5$, the operator $S_{\lambda}$ is bounded from $L^{p}\left(\mathbf{R}^{d} \times \mathbf{R}\right) \rightarrow L^{p}\left(\mathbf{R}^{d} \times \mathbf{R}\right)$ for $\lambda>\lambda^{*}(p), 2+\frac{4}{d}<p<\frac{8 d^{2}+2 d-6}{4 d^{2}-11 d-3}=2+\frac{6}{d-3}-\frac{6}{(4 d+1)(d-3)}$.

\section{Proof}

We begin by defining two new operators, $S_{\delta}$ and $C_{\delta}$, related to spherical means and the cone multiplier respectively:

$$
\begin{aligned}
\widehat{S_{\delta} f}(\xi)= & \varphi\left(\delta^{-1}(1-|\xi|)\right) \hat{f}(\xi), \\
\widehat{C_{\delta} f}(\xi)= & \varphi\left(\delta^{-1} h(\xi)\right) \kappa\left(\left(1-\left|\xi_{d+1}\right|\right)\right) \hat{f}(\xi), \\
& \text { where } h(\xi)=1-\frac{\left|\xi^{\prime}\right|}{\left|\xi_{d+1}\right|} .
\end{aligned}
$$

By using a dyadic decomposition, it is easy to see that the study of spherical means and the cone multiplier can by reduced to the study of these two new operators. $\varphi$ is in the class $\Phi$, which consists of all functions in $\mathcal{C}_{0}^{\infty}$ with the following properties:

$$
\begin{aligned}
\text { support of } \varphi & \subset[0,2], \\
\left|\frac{\partial^{|\alpha|}}{\partial \xi^{\alpha}} \varphi\right| & \lesssim C \quad \text { for all }|\alpha| \leq d+2,
\end{aligned}
$$


where $\alpha=\left(\alpha_{1}, \ldots, \alpha_{d+1}\right)$ is a standard multi-index and $C$ is a fixed constant. $\kappa$ is a $\mathcal{C}_{0}^{\infty}$ function supported in $\left(-\frac{1}{2}, \frac{1}{2}\right)$. $\delta$ is assumed to be a small positive number. The conjectured sharp bounds for $S_{\delta}$ and $C_{\delta}$ are

$$
\begin{aligned}
\left\|S_{\delta} g\right\|_{L^{p}\left(\mathbf{R}^{d}\right)} & \lesssim \delta^{-\lambda}\|g\|_{L^{p}\left(\mathbf{R}^{d}\right)}, \\
\left\|C_{\delta} f\right\|_{L^{p}\left(\mathbf{R}^{d} \times \mathbf{R}\right)} \lesssim \delta^{-\lambda}\|f\|_{L^{p}\left(\mathbf{R}^{d}\right)} & \text { for all } \lambda>\lambda^{*}(p) .
\end{aligned}
$$

Note that throughout the exposition, we will be using the symbol $\lesssim$ to denote that one expression is less than the other up to a constant which depends only on fixed values such as dimension and constants $\epsilon$ which are fixed at the beginning of proofs.

We now make a preliminary decomposition, then group the pieces into four cases which capture the nature of the multiplier $m_{\lambda}=\left(1-\max \left\{\left|\xi^{\prime}\right|,\left|\xi_{d+1}\right|\right\}\right)_{+}^{\lambda}$. Choose $\varphi \in \mathcal{C}_{0}^{\infty}(\mathbf{R})$ so that the support of $\varphi$ is contained in $\left[\frac{1}{2}, 2\right], \varphi \neq 0$ on $\left[\frac{3}{4}, \frac{3}{2}\right]$ and $\sum_{j=2}^{\infty} \varphi\left(2^{j} t\right) \equiv 1$ for $t \in\left(0, \frac{1}{4}\right)$. Choose $\varphi_{1}$ so that $\varphi_{1}(t)+\sum_{j=2}^{\infty} \varphi\left(2^{j}(1-t)\right) \equiv 1$ for $t \in[0,1)$.

Let

$$
\begin{aligned}
& m_{j k}(\xi)=\varphi\left(2^{j}\left(1-\left|\xi^{\prime}\right|\right)\right) \varphi\left(2^{k}\left(1-\left|\xi_{d+1}\right|\right)\right) m_{\lambda}(\xi) \quad \text { for } j, k \geq 2, \\
& m_{1 k}(\xi)=\varphi_{1}\left(\left|\xi^{\prime}\right|\right) \varphi\left(2^{k}\left(1-\left|\xi_{d+1}\right|\right)\right) m_{\lambda}(\xi) \quad \text { for } \quad k \geq 2 \text {, } \\
& m_{j 1}(\xi)=\varphi\left(2^{j}\left(1-\left|\xi^{\prime}\right|\right)\right) \varphi_{1}\left(\left|\xi_{d+1}\right|\right) m_{\lambda}(\xi) \quad \text { for } \quad j \geq 2 \text {, } \\
& m_{11}(\xi)=\varphi_{1}\left(\left|\xi^{\prime}\right|\right) \varphi_{1}\left(\left|\xi_{d+1}\right|\right) m_{\lambda}(\xi) .
\end{aligned}
$$

Then, using the triangle inequality,

$$
\left\|m_{\lambda}\right\|_{M^{p}} \leq \sum_{k, j=1}^{\infty}\left\|m_{j k}(\xi)\right\|_{M^{p}}
$$

where $\left\|m_{\lambda}\right\|_{M^{p}}$ denotes the operator norm of $f \rightarrow \mathcal{F}^{-1}\left[m_{\lambda} \widehat{f}\right]$.

We divide the sum into four parts and deal with each of them separately. The four parts are:

Case (i) $j \geq k+2$ : When $j \geq k+2$ the support of $m_{j k}$ is near the "sides" of the cylinder, where $\left|\xi^{\prime}\right|=1$. In this region $m_{\lambda}=\left(1-\left|\xi^{\prime}\right|\right)^{\lambda}$, so one expects the multiplier to behave like spherical means in $\mathbf{R}^{d}$.

Case (ii) $k \geq j+2$ : The support of $m_{j k}$ is near the "top" of the cylinder, where $\left|\xi_{d+1}\right|=1$. In this region $m_{\lambda}=\left(1-\left|\xi_{d+1}\right|\right)^{\lambda}$ which is a simple multiplier.

Case (iii) $|k-j| \leq 1, k, j \neq 1$ : This region contains the set where $\left|\xi^{\prime}\right|=$ $\left|\xi_{d+1}\right|$ (except near the origin). This is the most interesting case, where we explore the relation between $m_{\lambda}$ and the cone multiplier.

Case (iv) $|k-j| \leq 1, k=1$ or $j=1$ : We simply use scaling to extend our results from the first three cases to the center of the cylinder.

2.1. Case (i) $j \geq k+2$, near $\left|\xi^{\prime}\right|=1$.

Lemma 2.1. Let $2 \leq p<\infty$ and $\lambda>\lambda^{*}(p)$. Assume $\left\|S_{\delta} f\right\|_{L^{p}\left(\mathbf{R}^{d}\right)} \lesssim \delta^{-\lambda}\|f\|_{L^{p}\left(\mathbf{R}^{d}\right)}$ with constant independent of the choice of $\varphi \in \Phi$. Then the following sum converges:

$$
\sum_{k=1}^{\infty} \sum_{j=k+2}^{\infty}\left\|m_{j k}\right\|_{M^{p}} .
$$

This sum represents all multiplier pieces where $j \geq k+2$. 
Proof. Fix a small $\epsilon>0$.

When $j \geq k+2 \geq 4$, we have $\rho(\xi)=\left|\xi^{\prime}\right|$, so

$$
m_{j k}(\xi)=\varphi\left(2^{j}\left(1-\left|\xi^{\prime}\right|\right)\right) \varphi\left(2^{k}\left(1-\left|\xi_{d+1}\right|\right)\right)\left(1-\left|\xi^{\prime}\right|\right)^{\lambda} .
$$

Note that

$$
\begin{array}{rlr}
\left\|m_{j k}\right\|_{M_{p}} & =\left\|S_{2^{-j}, d}^{\prime}\right\|_{L^{p}\left(\mathbf{R}^{d}\right) \rightarrow L^{p}\left(\mathbf{R}^{d}\right)}\left\|\widetilde{S}_{2^{-k}}\right\|_{L^{p}(\mathbf{R}) \rightarrow L^{p}(\mathbf{R})} \quad \text { where } \\
S_{2^{-j}, d}^{\prime} f & =\mathcal{F}^{-1}\left[\varphi\left(2^{j}\left(1-\left|\xi^{\prime}\right|\right)\right)\left(1-\left|\xi^{\prime}\right|\right)^{\lambda}\right] * f \quad \text { and } \\
\widetilde{S}_{2^{-k}} f & =\mathcal{F}^{-1}\left[\varphi\left(2^{k}\left(1-\left|\xi_{d+1}\right|\right)\right)\right] * f .
\end{array}
$$

We note that for $S_{2^{-j}, d}^{\prime}$ we can use our assumption with $\delta=2^{-j}$ as follows. Define $\widetilde{\varphi}(t)=t^{\lambda} \varphi(t)$. Then

$$
S_{2^{-j}, d}^{\prime} f=2^{-j \lambda} \mathcal{F}^{-1}\left[\widetilde{\varphi}\left(2^{j}\left(1-\left|\xi^{\prime}\right|\right)\right)\right] * f .
$$

Since results such as 10 require only that $\varphi$ is a $C_{0}^{\infty}$ function, we obtain the following resulting bound. Here we are also using the fact that since the operator $S_{2^{-j}, d}^{\prime}$ acts only on the first $d$ variables, leaving the $\xi_{d+1}$ variable independent, we need only investigate the corresponding operator on $\mathbf{R}^{d}$, for which we use the same name:

$$
\left\|S_{2^{-j}, d}^{\prime}\right\|_{L^{p}\left(\mathbf{R}^{d}\right) \rightarrow L^{p}\left(\mathbf{R}^{d}\right)} \lesssim 2^{-j \lambda} 2^{j\left(\lambda^{*}(p)+\epsilon\right)}, \quad p_{0} \leq p<\infty .
$$

The operator $\widetilde{S}_{2^{-k}}$ only acts on the last variable, so we consider the corresponding operator on $\mathbf{R}$. A simple change of variable reveals that the $L^{1}$-norm of the kernel $\mathcal{F}^{-1}\left[\varphi\left(2^{k}\left(1-\left|\xi_{d+1}\right|\right)\right)\right]$ is bounded independent of $k$. Using this fact and Young's inequality gives us the following:

$$
\begin{aligned}
\left\|\widetilde{S}_{2^{-k}} f\right\|_{L^{p}(\mathbf{R})} & =\left\|\mathcal{F}^{-1}\left[\varphi\left(2^{k}\left(1-\left|\xi_{d+1}\right|\right)\right)\right] * f\right\|_{L^{p}(\mathbf{R})} \\
& \leq\left\|\mathcal{F}^{-1}\left[\varphi\left(2^{k}(1-|\cdot|)\right)\right]\right\|_{L^{1}(\mathbf{R})}\|f\|_{L^{p}(\mathbf{R})} \\
& \lesssim\|f\|_{L^{p}(\mathbf{R})} .
\end{aligned}
$$

The same bounds for $j$ and $k$ such that $j \geq k+2=3$ can be derived in a similar way. The operator associated with the multiplier $\varphi_{1}\left(\left|\xi_{d+1}\right|\right)$, which replaces $\varphi\left(2^{k}\left(1-\left|\xi_{d+1}\right|\right)\right)$ in the above calculation when $k=1$, is an operator bounded for all $L^{p}$ with norm independent of $k$. The approach we used on $\varphi\left(2^{k}\left(1-\left|\xi_{d+1}\right|\right)\right)$ will easily show this. Now we can sum over the range $j \geq k+2$.

$$
\sum_{k=1}^{\infty} \sum_{j=k+2}^{\infty}\left\|m_{j k}\right\|_{M^{p}} \lesssim \sum_{k=1}^{\infty} \sum_{j=k+2}^{\infty} 2^{j\left(-\lambda+\lambda^{*}(p)+\epsilon\right)} .
$$

This sum converges if $\lambda>\lambda^{*}(p)+\epsilon$.

2.2. Case (ii) $k \geq j+2$, near $\left|\xi_{d+1}\right|=1$. In this case, we are near the "top" of the cylinder, where $\left|\xi_{d+1}\right|=1$. However, since this case includes pieces which are also near the "sides" (when $j \approx k$ and $j, k$ are large), we must still apply results for $S_{\delta}$ 
Lemma 2.2. Let $2 \leq p<\infty$ and $\lambda>\lambda^{*}(p)$. Assume $\left\|S_{\delta} f\right\|_{L^{p}\left(\mathbf{R}^{d}\right)} \lesssim \delta^{-\lambda}\|f\|_{L^{p}\left(\mathbf{R}^{d}\right)}$ with constant independent of the choice of $\varphi \in \Phi$. Then the following sum converges:

$$
\sum_{j=1}^{\infty} \sum_{k=j+2}^{\infty}\left\|m_{j k}\right\|_{M^{p}} .
$$

This sum represents all multiplier pieces where $k \geq j+2$.

Proof. Fix a small $\epsilon>0$.

When $k \geq j+2 \geq 4$, we have $\rho(\xi)=\left|\xi_{d+1}\right|$, so

$$
m_{j k}(\xi)=\varphi\left(2^{j}\left(1-\left|\xi^{\prime}\right|\right)\right) \varphi\left(2^{k}\left(1-\left|\xi_{d+1}\right|\right)\right)\left(1-\left|\xi_{d+1}\right|\right)^{\lambda} .
$$

Similarly to the $j \geq k+2$ case,

$$
\begin{array}{rlr}
\left\|m_{j k}\right\|_{M_{p}} & =\left\|S_{2^{-j}, d}\right\|_{L^{p}\left(\mathbf{R}^{d}\right) \rightarrow L^{p}\left(\mathbf{R}^{d}\right)}\left\|\widetilde{S^{\prime}}{ }_{2^{-k}}\right\|_{L^{p}(\mathbf{R}) \rightarrow L^{p}(\mathbf{R})} \quad \text { where } \\
S_{2^{-j}, d} f & =\mathcal{F}^{-1}\left[\varphi\left(2^{j}\left(1-\left|\xi^{\prime}\right|\right)\right)\right] * f \quad \text { and } \\
{\widetilde{S^{\prime}}}_{2^{-k}} f & =\mathcal{F}^{-1}\left[\varphi\left(2^{k}\left(1-\left|\xi_{d+1}\right|\right)\right)\left(1-\left|\xi_{d+1}\right|\right)^{\lambda}\right] * f .
\end{array}
$$

For $S_{2^{-j}, d}$ we can directly apply assumption with $\delta=2^{-j}$. Here, as in Case (i), we use the fact that $S_{2^{-j}, d}$ acts only on the first $d$ variables. We obtain the following bound:

$$
\left\|S_{2^{-j}, d}\right\|_{L^{p}\left(\mathbf{R}^{d}\right) \rightarrow L^{p}\left(\mathbf{R}^{d}\right)} \lesssim 2^{j\left(\lambda^{*}(p)+\epsilon\right)}, \quad p_{0} \leq p<\infty .
$$

For the operator ${\widetilde{S^{\prime}}}_{2^{-k}}$, we note that using $\widetilde{\varphi}$ as defined above, we get

$$
{\widetilde{S^{\prime}}}_{2^{-k}} f=2^{-k \lambda} \mathcal{F}^{-1}\left[\widetilde{\varphi}\left(2^{k}\left(1-\left|\xi_{d+1}\right|\right)\right)\right] * f .
$$

We use the exact same reasoning as with $\widetilde{S}_{2^{-k}}$ in Case (i), replacing $\varphi$ with $\widetilde{\varphi}$ and noting the extra $2^{-k \lambda}$ gain. We now insert these bounds into our sum:

$$
\sum_{j=1}^{\infty} \sum_{k=j+2}^{\infty}\left\|m_{j k}\right\|_{M^{p}} \lesssim \sum_{j=1}^{\infty} \sum_{k=j+2}^{\infty} 2^{j\left(\lambda^{*}(p)+\epsilon\right)} 2^{-k \lambda} .
$$

This sum also converges if $\lambda>\lambda^{*}(p)+\epsilon$.

2.3. Case (iii) $|k-j| \leq 1, k, j \neq 1$, near $\left|\xi^{\prime}\right|=\left|\xi_{d+1}\right|$. In this region, we use the cone multiplier techniques in $\mathbf{R}^{d} \times \mathbf{R}$ to analyze the multipliers $m_{j k}$. We begin by subtracting a simpler multiplier in order to make our multiplier zero on the cone (where it is non-smooth). We then decompose the multiplier dyadically with respect to distance from the cone. This decomposition is done in detail in 2.3.3.

After decomposing, our pieces will not quite be cones, but rather "truncated cones". In the $\xi_{d+1}$ variable, our pieces will be supported in an interval of size about $2^{-j}$ due to the $\varphi\left(2^{k}\left(1-\left|\xi_{d+1}\right|\right)\right.$ ) (recall $k \approx j$ in Case (iii)). This is in contrast to the normal cone multiplier, where the multiplier is supported in an interval of size about 1 in the $\xi_{d+1}$ variable (see line (2.2)). These truncated cones will capture the effects of the increasing non-smoothness along the cone, which was alluded to earlier in the introduction.

We develop two bounds on these pieces. The first will be stronger for $p$ near $2+\frac{2}{d-1}$, where $\lambda^{*}(p)=0$. The second will be stronger for $p$ near $2+\frac{6}{d-3}$, where $\lambda^{*}(p)=1$. Recall that due to the relationship between our multiplier and the cone, the operator will be unbounded when $\lambda^{*}(p)>1$. 


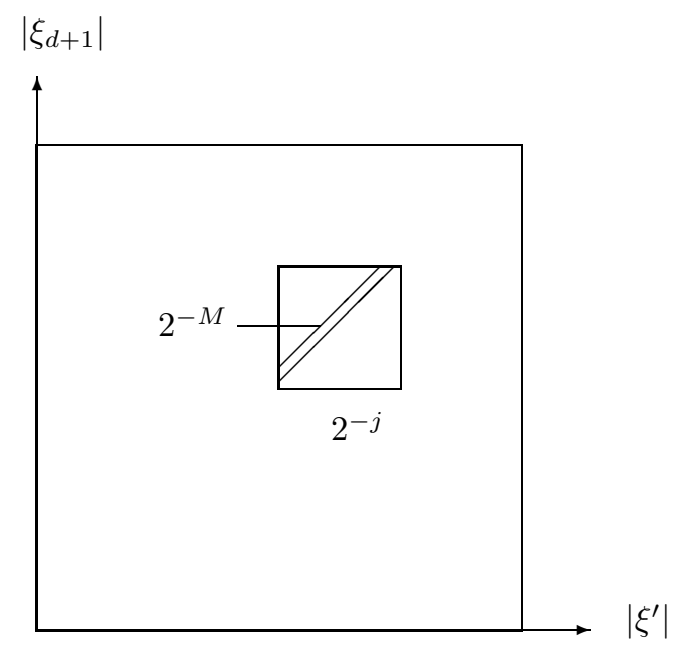

Figure 2. Support of the decomposition pieces

2.3.1. $\lambda^{*}(p)$ near 0 . Suppose $j \geq 2$. Let $\varphi_{j}$ be a function in $\mathcal{C}_{0}^{\infty}\left(\mathbf{R}^{d} \times \mathbf{R}\right)$ with the following properties:

$$
\begin{aligned}
\operatorname{supp}\left(\varphi_{j}\right) \subset \quad & \left\{\xi \in \mathbf{R}^{d} \times \mathbf{R}, \frac{1}{100}<2^{j}\left(1-\left|\xi^{\prime}\right|\right)<100\right. \\
& \text { and } \left.\frac{1}{100}<2^{j}\left(1-\left|\xi_{d+1}\right|\right)<100\right\}, \\
\left|\frac{\partial^{|\alpha|}}{\partial \xi^{\alpha}} \varphi_{j}(\xi)\right| \leq & C_{\varphi} 2^{|\alpha| j} \quad \text { for all }|\alpha| \leq d+2,
\end{aligned}
$$

where $C_{\varphi}$ is independent of $j$ and $\xi$.

We study the multiplier $\varphi\left(2^{M} h(\cdot)\right) \varphi_{j}$, where $h(\xi)=1-\frac{\left|\xi^{\prime}\right|}{\left|\xi_{d+1}\right|}$ as defined in line (2.2) and in the introduction. The decomposition in 2.3 .3 will reduce Case (iii) to multipliers of this type. The $\varphi_{j}$ term is supported in a ring a distance $2^{-j}$ from both the top and sides of the cylinder. It can be thought of as the result of rotating a cube with sidelength $2^{-j}$ around the $\xi_{d+1}$ axis. The $\varphi\left(2^{M} h(\cdot)\right)$ term is supported a distance $2^{-M}$ from the light cone. Together these are supported in a truncated cone of thickness $2^{-M}$ and "height" $2^{-j}$ in $\xi_{d+1}$ (see Figure 2).

For the following proposition we introduce an equispaced cutoff $\chi$. Let $\chi \in \mathcal{C}_{0}^{\infty}$, supp $\chi \subset(-1,1)$, and $\sum_{N=-\infty}^{\infty} \chi(\cdot-N) \equiv 1$.

Proposition 2.3. Let $2 \leq p_{0} \leq p_{c}<\infty$ and $\varphi \in \Phi$. Assume we have the following bounds:

$$
\begin{gathered}
\left\|\varphi\left(2^{M} h(\cdot)\right) \chi\left(2^{M(1+\epsilon)}\left((\cdot)_{d+1}-\frac{\nu}{2^{M(1+\epsilon)}}\right)\right) \varphi_{j}\right\|_{M^{p_{0}}} \\
\leq C_{1} C_{\varphi} 2^{M\left(\lambda^{*}\left(p_{0}\right)+\epsilon\right)}, \\
\left\|\varphi\left(2^{M} h(\cdot)\right) \varphi_{j}\right\|_{M^{p_{c}}} \leq C_{\varphi} C_{2} 2^{M\left(\lambda^{*}\left(p_{c}\right)+\epsilon\right)},
\end{gathered}
$$

where $\nu$ is any integer, and $C_{1}$ and $C_{2}$ are independent of $M, j$, and $\varphi_{j}$. 
Then for $p_{0} \leq p \leq p_{c}<\infty$,

$$
\begin{aligned}
\left\|\varphi\left(2^{M} h(\cdot)\right) \varphi_{j}\right\|_{M^{p}} \lesssim & C_{\varphi} 2^{(M-j)\left(\frac{\frac{1}{p}-\frac{1}{p_{c}}}{\frac{1}{p_{0}}-\frac{1}{p_{c}}}\right)\left(1-\frac{2}{p_{0}}\right)} 2^{M\left(\lambda^{*}(p)+\epsilon\right)} \\
& \text { for } M \geq j .
\end{aligned}
$$

In this proposition, (2.15) is a bound on the multiplier $\varphi\left(2^{M} h(\cdot)\right) \varphi_{j}$ truncated to a height of $2^{-M(1+\epsilon)}$. We then scale this bound to a useful bound on $\varphi\left(2^{M} h(\cdot)\right) \varphi_{j}$.

Proof. We begin by decomposing the support of $\varphi\left(2^{M} h(\cdot)\right) \varphi_{j}$ into rectangular "plates" in the standard way, as in [12. First, choose $\psi_{l} \in \mathcal{C}_{0}^{\infty}\left(\mathbf{R}^{d}\right), l=0,1, \ldots, L$, such that the support of $\psi_{l}$ has angular width less than $\frac{\pi}{4}, \sum_{j=0}^{L} \psi_{l} \equiv 1$ and $L$ depends only on $d$. We assume $\psi_{0}$ is supported in the region centered at the $\xi_{d}$-axis and we now investigate $\psi_{0}\left(\xi^{\prime}\right) \varphi_{j}(\xi)$. Bounds for $\psi_{l}\left(\xi^{\prime}\right) \varphi_{j}(\xi), l=1,2, \ldots, L$, follow by symmetry:

$$
\begin{gathered}
S_{M}=\left\{\mathbf{N}=\left\{N_{1}, \ldots, N_{d-1}\right\} \in \mathbf{Z}^{d-1}:-2^{\frac{M}{2}} \leq N_{n} \leq 2^{\frac{M}{2}}, 1 \leq n \leq d-1\right\} \\
\Phi_{M, \mathbf{N}}(\xi)=\varphi\left(2^{M} h(\xi)\right) \prod_{n=1}^{d-1} \chi\left(2^{\frac{M}{2}}\left(\frac{\xi_{n}}{\xi_{d+1}}-\frac{N_{n}}{2^{\frac{M}{2}}}\right)\right) \psi_{0}\left(\xi^{\prime}\right) \\
\varphi\left(2^{M} h(\xi)\right) \varphi_{j}(\xi) \psi_{0}\left(\xi^{\prime}\right)=\sum_{\mathbf{N} \in S_{M}} \Phi_{M, \mathbf{N}}(\xi) \varphi_{j}(\xi)
\end{gathered}
$$

We may assume that $M \geq j-10$ (or $M \geq 0$ if $j-10<0$ ) since

$$
\operatorname{supp}\left[\varphi\left(2^{M} h(\cdot)\right)\right] \cap \operatorname{supp} \varphi_{j}=\emptyset \quad \text { if } M<j-10 .
$$

Note that $\sum_{\mathbf{N}} \Phi_{M, \mathbf{N}} \varphi_{j}$ is supported on a cone truncated to height $2^{-j}$. Also, the terms $\Phi_{M, \mathbf{N}} \varphi_{j}$ are supported in rectangular plates lying against the cone with dimensions

$$
2^{-M} \times \underbrace{2^{-\frac{M}{2}} \times \cdots \times 2^{-\frac{M}{2}}}_{d-1 \text { times }} \times 2^{-j} .
$$

We further decompose in $\xi_{d+1}$, dividing the decomposed cone into cones truncated to a height $2^{-\frac{M}{c_{h}}}$. The constant $c_{h}$ determines the size of the decomposition. In proving Proposition 2.3, we will decompose using $c_{h}=1-\epsilon$. This decomposition is in some sense excessively severe but it allows the multiplier to be related to spherical means in $\mathbf{R}^{d}$ (see Lemma 2.12). Later in 2.3.2, Proposition 2.5 will use a much more efficient decomposition with $c_{h}=2$ :

$$
\begin{aligned}
\chi_{\nu}\left(\xi_{d+1}\right) & =\chi\left(2^{\frac{M}{c_{h}}}\left(\xi_{d+1}-\frac{\nu}{2^{\frac{M}{c_{h}}}}\right)\right), \\
\Phi_{M, \mathbf{N}}(\xi) & =\sum_{\nu=-2^{\frac{M}{c_{h}}}}^{2^{\frac{M}{c_{h}}}} \chi_{\nu}\left(\xi_{d+1}\right) \Phi_{M, \mathbf{N}}(\xi) \\
& =\sum_{\nu=-2^{\frac{M}{c_{h}}}}^{2^{\frac{M}{c_{h}}}} \Phi_{M, \mathbf{N}, \nu}(\xi) .
\end{aligned}
$$


For now, we only use the vertical decomposition in $\nu$ and not the plates decomposition in $\mathbf{N}$. Later, however, the plates decomposition will be essential in determining estimates in the form of (2.15) and (2.16).

We use the following lemma to take advantage of the equispaced aspect of the vertical decomposition.

Lemma 2.4. Let $\tilde{\chi}$ be defined so that $\tilde{\chi} \equiv 1$ on $\operatorname{supp}(\chi) \subset(-1,1)$ and $\tilde{\chi} \equiv 0$ outside $\left(\frac{-3}{2}, \frac{3}{2}\right)$. Let $\widetilde{\chi}_{\nu}\left(\xi_{d+1}\right)=\tilde{\chi}\left(2^{\frac{M}{c_{h}}}\left(\xi_{d+1}-\frac{\nu}{2^{\frac{M}{c_{h}}}}\right)\right)$, and let $f_{\nu}=\mathcal{F}^{-1}\left[\widetilde{\chi}_{\nu} * f\right]$. Let $\mathcal{V}$ be a finite interval in the integers $\mathbf{Z}$. Then for $2 \leq p \leq \infty, \frac{1}{p^{\prime}}+\frac{1}{p}=1$ and $f \in L^{p}$,

$$
\begin{aligned}
\left\|\sum_{\nu \in \mathcal{V}} \mathcal{F}^{-1}\left[\chi_{\nu}\right] * f\right\|_{L^{p}} & \lesssim\left(\sum_{\nu \in \mathcal{V}}\left\|\mathcal{F}^{-1}\left[\chi_{\nu}\right] * f\right\|_{L^{p}}^{p^{\prime}}\right)^{\frac{1}{p^{\prime}}} \\
& =\left(\sum_{\nu \in \mathcal{V}}\left\|\mathcal{F}^{-1}\left[\chi_{\nu}\right] * f_{\nu}\right\|_{L^{p}}^{p^{\prime}}\right)^{\frac{1}{p^{\prime}}}
\end{aligned}
$$

and

$$
\left(\sum_{\nu \in \mathcal{V}}\left\|\mathcal{F}^{-1}\left[\chi_{\nu}\right] * f_{\nu}\right\|_{L^{p}}^{p^{\prime}}\right)^{\frac{1}{p^{\prime}}} \lesssim(\operatorname{card}(\mathcal{V}))^{\left(1-\frac{2}{p}\right)}\|f\|_{L^{p}}
$$

This lemma is easily verified for $p=2$ using Plancherel's theorem.

The first inequality in the lemma follows by interpolation and duality from the $p=2$ bound and the following $p=1$ bound (triangle inequality):

$$
\begin{aligned}
\left\|\sum_{\nu} \mathcal{F}^{-1}\left[\chi_{\nu}\right] * f\right\|_{L^{1}} & \leq \sum_{\nu}\left\|\mathcal{F}^{-1}\left[\chi_{\nu}\right] * f\right\|_{L^{1}} \\
& =\left(\sum_{\nu}\left\|\mathcal{F}^{-1}\left[\chi_{\nu}\right] * f\right\|_{L^{1}}^{1}\right)^{1} .
\end{aligned}
$$

For the second inequality in the lemma, the $L^{\infty}$ bound follows from the uniform (in $\nu$ ) boundedness of the operators $\mathcal{F}^{-1}\left[\chi_{\nu}\right] * f$. The lemma follows by interpolation:

$$
\begin{aligned}
\left(\sum_{\nu \in \mathcal{V}}\left\|\mathcal{F}^{-1}\left[\chi_{\nu}\right] * f_{\nu}\right\|_{L^{\infty}}\right) & =\left(\sum_{\nu \in \mathcal{V}}\left\|\mathcal{F}^{-1}\left[\chi_{\nu}\right] * f\right\|_{L^{\infty}}\right) \\
& \lesssim\left(\sum_{\nu \in \mathcal{V}}\|f\|_{L^{\infty}}\right) \\
& =(\operatorname{card}(\mathcal{V}))\|f\|_{L^{\infty}} .
\end{aligned}
$$


Using this lemma, we obtain the following $L^{p_{0}}$ bound. Here $\mathcal{V}$ will consist of those $\nu$ such that the support of $\chi_{\nu}$ intersects the support of $\varphi_{j}$ :

$$
\begin{aligned}
\| \mathcal{F}^{-1} & {\left[\varphi\left(2^{M} h(\cdot)\right) \varphi_{j} \psi_{0}(\cdot)\right] * f \|_{L^{p_{0}}} } \\
= & \left\|\sum_{\nu \in \mathcal{V}} \sum_{\mathbf{N} \in S_{M}} \mathcal{F}^{-1}\left[\Phi_{M, \mathbf{N}, \nu} \varphi_{j}\right] * f_{\nu}\right\|_{L^{p_{0}}} \\
& \lesssim\left(\sum_{\nu \in \mathcal{V}}\left\|\mathcal{F}^{-1}\left[\sum_{\mathbf{N} \in S_{M}} \Phi_{M, \mathbf{N}, \nu} \varphi_{j}\right] * f_{\nu}\right\|_{L^{p_{0}}}^{p_{0}^{\prime}}\right)^{\frac{1}{p_{0}^{\prime}}} \\
& \lesssim C_{\varphi} 2^{M\left(\lambda^{*}\left(p_{0}\right)+\epsilon\right)}\left(\sum_{\nu \in \mathcal{V}}\left\|f_{\nu}\right\|_{L^{p_{0}}}^{p^{\prime}}\right)^{\frac{1}{p_{0}^{\prime}}} \\
& \lesssim 2^{M\left(\lambda^{*}\left(p_{0}\right)+\epsilon\right)} t_{M j}^{\left(1-\frac{2}{p_{0}}\right)}\|f\|_{L^{p_{0}}},
\end{aligned}
$$

where $\operatorname{card}(\mathcal{V})=t_{M j}$ and $t_{M j}$ is defined as follows:

$$
t_{M j} \lesssim \begin{cases}1 & \text { if } j-10 \leq M \leq c_{h} j, \\ 2^{\frac{M}{c_{h}}-j} & \text { if } M>c_{h} j .\end{cases}
$$

In this case, since $c_{h}=1-\epsilon$, we effectively have $t_{M j}=2^{M-j} 2^{M \epsilon}$. By interpolating between line (2.30) and line (2.16), we obtain the bounds of the proposition.

2.3.2. $\lambda^{*}(p)$ near 1. Proposition 2.3 is designed, with its severe decomposition in $\xi_{d+1}$, to allow us to use Bochner-Riesz results from $\mathbf{R}^{d}$. However, the inefficiency in this decomposition and Lemma 2.4 limit results to $p$ where $\lambda^{*}(p)$ is small. There is too much loss for the eventual sum in $M$ to converge if $\lambda^{*}(p)$ is near 1. We introduce Proposition 2.5, which will not apply for $\lambda^{*}(p)$ small, but which will significantly improve upon the $\left(\frac{\frac{1}{p}-\frac{1}{p_{c}}}{\frac{1}{p_{0}}-\frac{1}{p_{c}}}\right)\left(1-\frac{2}{p_{0}}\right)$ loss in Proposition 2.3 for $\lambda^{*}(p)$ near 1.

We obtain a result for truncated cones by combining a square function estimate with a result for the Nikodym maximal function for light rays. For the square function estimate we can adapt Bourgain's argument 1 or in $\mathbf{R}^{2} \times \mathbf{R}$ there is a result of Mockenhaupt, Seeger and Sogge (MSS) 13]. Mockenhaupt, Seeger and Sogge [14 and Skarabot 17 have determined maximal function results, and in $\mathbf{R}^{2} \times \mathbf{R}$ there is a maximal function bound due to Córdoba 5 .

The Nikodym maximal function for light rays $M_{\delta} f$ can be defined as follows:

$$
\begin{aligned}
\tau_{\delta}(\theta)= & \delta \times \cdots \times \delta \times 1 \text { tube with long direction } \\
& \left(\xi^{\prime}, \xi_{d+1}\right)=(\theta, 1), \text { centered at the origin, } \theta \in S^{d-1}, \\
M_{\delta} f(x)= & \sup _{\theta \in S^{d-1}} \delta^{-(d)} \int_{\tau_{\delta}(\theta)}|f(x+y)| d y .
\end{aligned}
$$

This maximal function takes maximum of averages over tubes pointing in the direction of light rays ( $\frac{\pi}{4}$ from the horizontal). The conjectured bound for this maximal function is

$$
\left\|M_{\delta} f\right\|_{L^{p}\left(\mathbf{R}^{d} \times \mathbf{R}\right)} \lesssim \delta^{1-\frac{d}{p}}\|f\|_{L^{p}\left(\mathbf{R}^{d} \times \mathbf{R}\right)} .
$$


Proposition 2.5. Let $p_{0} \geq \frac{2(d+1)}{d-1}$ and let $\frac{1}{q_{0}}+\frac{2}{p_{0}}=1$ (we say $\left.q_{0}=\left(\frac{p_{0}}{2}\right)^{\prime}\right)$. Assume the following bound on the Nikodym maximal function for light rays:

$$
\left\|M_{\delta} f\right\|_{L^{q_{0}}} \lesssim \delta^{-a} \delta^{1-\frac{d}{q_{0}}-\epsilon}\|f\|_{L^{q_{0}}}, \quad a>0 .
$$

Also, assume that for some $p_{c}>p_{0}$,

$$
\left\|\varphi\left(2^{M} h(\cdot)\right) \varphi_{j}\right\|_{M^{p_{c}}} \leq C_{\varphi} C_{2} 2^{M\left(\lambda^{*}\left(p_{c}\right)+\epsilon\right)},
$$

independent of $\varphi \in \Phi\left(C_{\varphi}\right.$ is from the definition of $\varphi_{j}$ in 2.3.1). Then for $p_{0} \leq p \leq$ $p_{c}$,

$$
\begin{gathered}
\left\|\varphi\left(2^{M} h(\cdot)\right) \varphi_{j}\right\|_{M^{p}} \lesssim \quad C_{\varphi} 2^{\left(\left(\frac{M}{2}-j\right)\left(\frac{1}{2}-\frac{1}{p_{0}}\right)+M\left(\frac{a}{4}\right)\right)\left(\frac{\frac{1}{p}-\frac{1}{p_{c}}}{\frac{1}{p_{0}}-\frac{1}{p_{c}}}\right)} 2^{M\left(\lambda^{*}(p)+\epsilon\right)} \\
\quad \text { for } M \geq 2 j
\end{gathered}
$$

and

$$
\begin{gathered}
\left\|\varphi\left(2^{M} h(\cdot)\right) \varphi_{j}\right\|_{M^{p}} \lesssim \quad C_{\varphi} 2^{(M-j)\left(\frac{a}{2}\right)\left(\frac{\frac{1}{p}-\frac{1}{p_{c}}}{\frac{1}{p_{0}}-\frac{1}{p_{c}}}\right)} 2^{M\left(\lambda^{*}(p)+\epsilon\right)} \\
\quad \text { for } j \leq M \leq 2 j .
\end{gathered}
$$

Throughout the proof of Proposition 2.5 we will be using a decomposition in $\xi_{d+1}$ of height $2^{-\frac{M}{2}}$. Lemma 2.4 will apply with $c_{h}=2$. We set $c_{h}=2$ in the definitions of $\chi_{\nu}, t_{M j}, \Phi_{M, \mathbf{N}, \nu}$, etc.

Proof. To prove this proposition at $p_{0}$, we follow a standard strategy of first applying a square function estimate, followed by a maximal function, then returning from the square function back to $f$. For an example of this approach, one can see 4] where Córdoba proves the Bochner-Riesz conjecture in $\mathbf{R}^{2}$ in this way. We will require the following lemmas.

We begin with the square function estimate. We plan to replace the $L^{p}$-norm with a $L^{p}\left(l^{2}\right)$-norm over the terms $\mathcal{F}^{-1}\left[\Phi_{M, \mathbf{N}, \nu}\right] * f$. We do this by covering our truncated cone with $2^{-\frac{M}{2}}$-cubes and following the method of Bourgain 11. However, it is convenient to have each $\Phi_{M, \mathbf{N}, \nu}$ supported in a single cube, so we have to enlarge the cubes slightly.

Fix $M$. Note that the multiplier $\left(1-\max \left\{\left|\xi^{\prime}\right|,\left|\xi_{d+1}\right|\right\}\right)_{+}^{\lambda}$ is supported in a cube of radius 2 (it is smaller than this, but we take extra to be safe). Let $\{Q\}$ be a tiling of this support cube by cubes of sidelength $2^{-\frac{M}{2}}$. Choose $\widetilde{\chi}_{0} \in \mathcal{C}_{0}^{\infty}\left(\mathbf{R}^{d}\right)$ to be identically 1 on the unit cube and supported in the double of the unit cube. Let $\tilde{\chi}_{Q}\left(\xi^{\prime}\right)=\tilde{\chi}_{0}\left(C 2^{\frac{M}{2}}\left(\xi^{\prime}-\xi_{Q}^{\prime}\right)\right)$, where $\xi_{Q}^{\prime}$ is the center of $Q$. $C$ is chosen so that each $\Phi_{M, \mathbf{N}, \nu} \chi_{Q} \equiv 0$ for all but a finite number (independent of $\left.M, \mathbf{N}, \nu\right)$ of $Q$, and there is at least one $Q$, denoted $Q_{\mathbf{N}}$, such that $\widetilde{\chi}_{Q_{\mathbf{N}}} \equiv 1$ on the support of $\Phi_{M, \mathbf{N}, \nu}$. Let $\chi_{\mathbf{N}, \nu}=\widetilde{\chi}_{Q_{\mathbf{N}}}\left(\xi^{\prime}\right) \widetilde{\chi}_{\nu}\left(\xi_{d+1}\right)$.

Lemma 2.6. Let $p \geq \frac{2(d+1)}{d-1}$. Let

$$
\mathcal{Q}=\left\{(\mathbf{N}, \nu): \operatorname{supp}\left(\chi_{\mathbf{N}, \nu}\right) \cap \operatorname{supp}\left(\varphi\left(2^{M} h(\cdot)\right) \varphi_{j}\right) \neq \emptyset .\right.
$$


Then

(2.38)

$$
\begin{aligned}
\left\|\sum_{(\mathbf{N}, \nu) \in \mathcal{Q}} \mathcal{F}^{-1}\left[\chi_{\mathbf{N}, \nu}\right] * f\right\|_{L^{p}} \\
\quad \lesssim(\operatorname{card}(\mathcal{V}))^{\left(\frac{1}{2}-\frac{1}{p}\right)} 2^{\left(\frac{M}{2}\right)\left(\lambda^{*}(p)+\epsilon\right)}\left\|\left(\sum_{(\mathbf{N}, \nu) \in \mathcal{Q}}\left|\mathcal{F}^{-1}\left[\chi_{\mathbf{N}, \nu}\right] * f\right|^{2}\right)^{\frac{1}{2}}\right\|_{L^{p}} .
\end{aligned}
$$

Here $\mathcal{V}=\{\nu \in \mathcal{Z}:(\mathbf{N}, \nu) \in \mathcal{Q}$ for some $\mathbf{N}\}$. In the future, we denote $\mathcal{F}^{-1}\left[\chi_{\mathbf{N}, \nu}\right] *$ $f=f_{\mathbf{N}, \nu}$.

Note that in the above lemma, it is important that the sum runs over only those cubes which intersect the truncated cone where the multiplier piece is supported. The proof will follow in Section 3.1.

Once we have decomposed the operator, the pieces of the multiplier have convolution kernels which can be bounded by the simple operators, as detailed in the following lemma.

Lemma 2.7. For fixed $\mathbf{N} \in \mathbf{Z}^{d-1}$, define

$$
\begin{array}{rll}
\rho(\xi) & = & \left|\xi^{\prime}\right|, \\
\tilde{\xi}_{0} & = & \text { center of } \operatorname{supp}\left(\Phi_{M, \mathbf{N}, \nu} \varphi_{j}\right), \\
\mathbf{u}_{d} & =\frac{1}{\sqrt{2}}\left(\begin{array}{c}
\nabla \rho\left(\tilde{\xi}_{0}\right) \\
-1
\end{array}\right), \\
\mathbf{u}_{d+1} & =\frac{1}{\sqrt{2}}\left(\begin{array}{c}
\nabla \rho\left(\tilde{\xi}_{0}\right) \\
1
\end{array}\right), \\
\left\{\mathbf{u}_{i}\right\}_{i=1}^{d-1} \quad \text { span } & \left(\begin{array}{c}
\nabla \rho\left(\tilde{\xi}_{0}\right)^{\perp} \\
0
\end{array}\right) .
\end{array}
$$

Then for every choice of integers $p_{1}, \ldots, p_{d+1} \geq 0$,

$$
\begin{aligned}
\left|\mathcal{F}^{-1}\left[\Phi_{M, \mathbf{N}, \nu} \varphi_{j}\right]\right| \lesssim & 2^{-\frac{M}{2} d} 2^{-M} \prod_{i=1}^{d-1}\left(\frac{1}{\left(1+\left|2^{-\frac{M}{2}} \mathbf{u}_{i} \cdot x\right|\right)^{p_{i}}}\right) \\
& \cdot \frac{1}{\left(1+\left(\left|2^{-M} \mathbf{u}_{d} \cdot x\right|\right)^{p_{d}}\right.} \frac{1}{\left(1+\left(\left|2^{-\frac{M}{2}} \mathbf{u}_{d+1} \cdot x\right|\right)^{p_{d+1}}\right.} \\
\lesssim & \sum_{m=0}^{\infty} 2^{-m} \frac{1}{\left|R_{m}^{\mathbf{N}}\right|} \chi_{R_{m}^{\mathrm{N}}}(x)
\end{aligned}
$$

where $R_{m}^{\mathbf{N}}=\left\{x:\left|\mathbf{u}_{d} \cdot x\right| \leq 2^{m} 2^{M},\left|\mathbf{u}_{d+1} \cdot x\right| \leq 2^{m} 2^{\frac{M}{2}},\left|\mathbf{u}_{i} \cdot x\right| \leq 2^{m} 2^{\frac{M}{2}}\right.$ for $1 \leq i \leq$ $d-1\}$. Note that this bound implies that $\left\|\mathcal{F}^{-1}\left[\Phi_{M, \mathbf{N}, \nu} \varphi_{j}\right]\right\|_{L^{1}}<C$ independent of $M, \mathbf{N}, \nu$ and $j$.

The proof of Lemma 2.7 will be presented in Section 3.2

Lemma 2.8. Let $p \geq 2$. Then

$$
\left\|\left(\sum_{\mathbf{N}, \nu}\left|\mathcal{F}^{-1}\left[\chi_{\mathbf{N}, \nu}\right] * f\right|^{2}\right)^{\frac{1}{2}}\right\|_{L^{p}} \lesssim\|f\|_{L^{p}} .
$$


This Littlewood-Paley type estimate will allow us to return from a square function back to a regular norm. Lemma 2.8 is due to Carleson. Proofs can be found in [5] and [16. With this formulation, the lemma is relatively easy to verify since the decomposition is equally spaced and the cutoff functions are merely translations of each other.

We now proceed with the calculation. We begin with the $p_{0}$ bound, assuming $M \geq 2 j$,

$$
\begin{gathered}
\left\|\sum_{\mathbf{N}, \nu} \mathcal{F}^{-1}\left[\Phi_{M, \mathbf{N}, \nu} \varphi_{j}\right] * f\right\|_{L^{p_{0}}}=\left\|\sum_{\mathbf{N}, \nu} \mathcal{F}^{-1}\left[\Phi_{M, \mathbf{N}, \nu} \varphi_{j}\right] * f_{\mathbf{N}, \nu}\right\|_{L^{p_{0}}} \\
\vdots 2^{\left(\frac{M}{2}-j\right)\left(\frac{1}{2}-\frac{1}{p_{0}}\right)} 2^{\left(\frac{M}{2}\right)\left(\lambda^{*}\left(p_{0}\right)+\epsilon\right)}\left\|\left(\sum_{\mathbf{N}, \nu}\left|\mathcal{F}^{-1}\left[\Phi_{M, \mathbf{N}, \nu} \varphi_{j}\right] * f_{\mathbf{N}, \nu}\right|^{2}\right)^{\frac{1}{2}}\right\|_{L^{p_{0}}} \\
\left\|\left(\sum_{\mathbf{N}, \nu}\left|\mathcal{F}^{-1}\left[\Phi_{M, \mathbf{N}, \nu} \varphi_{j}\right] * f_{\mathbf{N}, \nu}\right|^{2}\right)^{\frac{1}{2}}\right\|_{L^{p_{0}}}^{2} \\
=\left(\left.\left.\int\left|\sum_{\mathbf{N}, \nu}\right| \mathcal{F}^{-1}\left[\Phi_{M, \mathbf{N}, \nu} \varphi_{j}\right] * f_{\mathbf{N}, \nu}\right|^{2}\right|^{\frac{p_{0}}{2}}\right)^{\frac{2}{p_{0}}} \\
=\sup _{\|\omega\|_{\left(\frac{p_{0}}{2}\right)^{\prime}} \leq 1} \int\left(\sum_{\mathbf{N}, \nu}\left|\mathcal{F}^{-1}\left[\Phi_{M, \mathbf{N}, \nu} \varphi_{j}\right] * f_{\mathbf{N}, \nu}\right|^{2}\right) \omega .
\end{gathered}
$$

Let $\|\omega\|_{\left(\frac{p_{0}}{2}\right)^{\prime}} \leq 1$. Then

$$
\begin{aligned}
& \int\left(\sum_{\mathbf{N}, \nu}\left|\mathcal{F}^{-1}\left[\Phi_{M, \mathbf{N}, \nu} \varphi_{j}\right] * f_{\mathbf{N}, \nu}\right|^{2}\right) \omega \\
& \lesssim \sum_{m=0}^{\infty} 2^{-m} \int \sum_{\mathbf{N}, \nu}\left|\frac{1}{\left|R_{m}^{\mathbf{N}}\right|} \chi_{R_{m}^{\mathbf{N}}} * f_{\mathbf{N}, \nu}\right|^{2} \omega \\
& \int \sum_{\mathbf{N}, \nu} \mid \frac{1}{\left|R_{m}^{\mathbf{N}}\right|} \chi_{\left.R_{m}^{\mathbf{N}} * f_{\mathbf{N}, \nu}\right|^{2} \omega} \\
& \leq \int \sum_{\mathbf{N}, \nu}\left(\int \frac{1}{\left|R_{m}^{\mathbf{N}}\right|} \chi_{R_{m}^{\mathbf{N}}}(y) d y\right) \\
& \cdot\left(\int \frac{1}{\left|R_{m}^{\mathbf{N}}\right|} \chi_{R_{m}^{\mathbf{N}}}(x-y)\left|f_{\mathbf{N}, \nu}(y)\right|^{2} d y\right) \omega(x) d x
\end{aligned}
$$




$$
\begin{aligned}
& \leq \int \sum_{\mathbf{N}, \nu}\left|f_{\mathbf{N}, \nu}(y)\right|^{2} \int \frac{1}{\left|R_{m}^{\mathbf{N}}\right|} \chi_{R_{m}^{\mathbf{N}}}(x-y) \omega(x) d x d y \\
& \leq \int \sum_{\mathbf{N}, \nu}\left|f_{\mathbf{N}, \nu}(y)\right|^{2} M_{2^{-\frac{M}{2}}} \omega(y) d y \\
& \leq\left\|\left(\sum_{\mathbf{N}, \nu}\left|f_{\mathbf{N}, \nu}\right|^{2}\right)^{\frac{1}{2}}\right\|_{L^{p_{0}}}^{2}\left\|M_{2^{-\frac{M}{2}}} \omega\right\|_{L^{\left(\frac{p_{0}}{2}\right)^{\prime}}} \\
& \lesssim\|f\|_{L^{p_{0}}}^{2} 2^{M\left(\lambda^{*}\left(p_{0}\right)+\epsilon\right)} 2^{\frac{M}{2} a}, \\
&\left\|\sum_{\nu \in \mathcal{V}} \sum_{\mathbf{N} \in S_{M}} \mathcal{F}^{-1}\left[\Phi_{M, \mathbf{N}, \nu} \varphi_{j}\right] * f\right\|_{L^{p_{0}}} \\
& \lesssim 2^{\left(\frac{M}{2}-j\right)\left(\frac{1}{2}-\frac{1}{p_{0}}\right)} 2^{M\left(\lambda^{*}\left(p_{0}\right)+\epsilon\right)} 2^{\frac{M}{4} a}\|f\|_{L^{p_{0}}} .
\end{aligned}
$$

Line (2.46) is an application of the Cauchy-Schwartz inequality. In line (2.50), we use the fact that $\frac{1}{2}\left(1-\frac{d}{\left(\frac{p}{2}\right)^{\prime}}\right)=-\lambda^{*}(p)$.

(2.36) follows by interpolation between (2.51) and the assumed bound at $p_{c}$.

Now assume $j \leq M \leq 2 j$. Here we must change our decomposition slightly. We still wish to decompose into square plates (so that the maximal function is as efficient as possible); however, now the "height" of our truncated cone is less than $2^{-\frac{M}{2}}$. Instead we decompose into plates which are $2^{-M}$ thick and $2^{-j}$ in every other direction,

$$
\begin{gathered}
\widetilde{S}_{M}=\left\{\mathbf{N}=\left\{N_{1}, \ldots, N_{d-1}\right\}:-2^{j} \leq N_{n} \leq 2^{j} \quad \text { for all } n=1, \ldots, d-1\right\} \\
\widetilde{\Phi}_{M, \mathbf{N}}(\xi)=\varphi\left(2^{M} h(\xi)\right) \prod_{n=1}^{d-1} \chi\left(2^{j}\left(\frac{\xi_{n}}{\xi_{d+1}}-\frac{N_{n}}{2^{j}}\right)\right) \psi_{0}\left(\xi^{\prime}\right), \\
\varphi\left(2^{M} h(\xi)\right) \varphi_{j}(\xi) \psi_{0}\left(\xi^{\prime}\right)=\sum_{\mathbf{N} \in \widetilde{S}_{M}} \widetilde{\Phi}_{M, \mathbf{N}}(\xi) \varphi_{j}(\xi) .
\end{gathered}
$$

Note that the terms $\widetilde{\Phi}_{M, \mathbf{N}} \varphi_{j}$ are supported in rectangular plates lying against the cone with dimensions

$$
2^{-M} \times \underbrace{2^{-j} \times \cdots \times 2^{-j}}_{d \text { times }}
$$

We use slight variations on Lemmas 2.6 and 2.7

Similar to the $\chi_{Q_{\mathrm{N}}}$ terms from Lemma 2.6 which defined a decomposition into cubes of side $2^{-\frac{M}{2}}$ on the Fourier side, we now define new functions $\chi_{\mathbf{N}}^{\prime}$. These new functions are defined in the same way, except for cubes of side $2^{-j}$. Note that since the truncated cone is just $2^{-j}$ high, we do not need the $\nu$ decomposition in $\xi_{d+1}$.

Lemma 2.9. Let $p \geq \frac{2(d+1)}{d-1}$. Let

$$
\mathcal{Q}^{\prime}=\left\{\mathbf{N}: \operatorname{supp}\left(\chi_{\mathbf{N}}\right) \cap \operatorname{supp}\left(\varphi\left(2^{M} h(\cdot)\right) \varphi_{j}\right) \neq \emptyset .\right.
$$

Then

$$
\left\|\sum_{\mathbf{N} \in \mathcal{Q}^{\prime}} \mathcal{F}^{-1}\left[\chi_{\mathbf{N}}^{\prime}\right] * f\right\|_{L^{p}} \lesssim 2^{j\left(\lambda^{*}(p)+\epsilon\right)}\left\|\left(\sum_{\mathbf{N} \in \mathcal{Q}^{\prime}}\left|\mathcal{F}^{-1}\left[\chi_{\mathbf{N}}^{\prime}\right] * f\right|^{2}\right)^{\frac{1}{2}}\right\|_{L^{p}} .
$$

In the future, we denote $\mathcal{F}^{-1}\left[\chi_{\mathbf{N}}^{\prime}\right] * f=\widetilde{f}_{\mathbf{N}}$. 
The proof is identical to that of Lemma 2.6 in Section 3.1 .

Lemma 2.10. For fixed $\mathbf{N} \in \mathbf{Z}^{d-1}$, define

$$
\begin{array}{rll}
\rho(\xi) & =\left|\xi^{\prime}\right|, \\
\tilde{\xi}_{0} & =\text { center of } \operatorname{supp}\left(\widetilde{\Phi}_{M, \mathbf{N}, \nu} \varphi_{j}\right), \\
\mathbf{u}_{d} & =\frac{1}{\sqrt{2}}\left(\begin{array}{c}
\nabla \rho\left(\tilde{\xi}_{0}\right) \\
-1
\end{array}\right), \\
\mathbf{u}_{d+1} & =\frac{1}{\sqrt{2}}\left(\begin{array}{c}
\nabla \rho\left(\tilde{\xi}_{0}\right) \\
1
\end{array}\right), \\
\left\{\mathbf{u}_{i}\right\}_{i=1}^{d-1} \quad \text { span } & \left(\begin{array}{c}
\nabla \rho\left(\tilde{\xi}_{0}\right)^{\perp} \\
0
\end{array}\right) .
\end{array}
$$

Assume $j \leq M \leq 2 j$. Then for every choice of integers $p_{1}, \ldots, p_{d+1} \geq 0$,

$$
\begin{aligned}
\left|\mathcal{F}^{-1}\left[\widetilde{\Phi}_{M, \mathbf{N}} \varphi_{j}\right]\right| \lesssim & 2^{-j d} 2^{-M} \prod_{i=1}^{d-1} \frac{1}{\left(1+\left|2^{-j} \mathbf{u}_{i} \cdot x\right|\right)^{p_{i}}} \\
& \cdot \frac{1}{\left(1+\left(\left|2^{-M} \mathbf{u}_{d} \cdot x\right|\right)^{p_{d}}\right.} \frac{1}{\left(1+\left(\left|2^{-j} \mathbf{u}_{d+1} \cdot x\right|\right)^{p_{d+1}}\right.} \\
\lesssim & \sum_{m=0}^{\infty} 2^{-m} \frac{1}{\left|\widetilde{R}_{m}^{\mathbf{N}}\right|} \chi_{\widetilde{R}_{m}^{\mathrm{N}}}(x)
\end{aligned}
$$

where $\widetilde{R}_{m}^{\mathbf{N}}=\left\{x:\left|\mathbf{u}_{d} \cdot x\right| \leq 2^{m} 2^{M},\left|\mathbf{u}_{d+1} \cdot x\right| \leq 2^{m} 2^{j},\left|\mathbf{u}_{i} \cdot x\right| \leq 2^{m} 2^{j}\right.$ for $\left.1 \leq i \leq d-1\right\}$.

The proof is the same as that of Lemma 2.7

Now we simply follow the proof of the $M \geq 2 j$ case, using these new lemmas. Note that the Nikodym maximal function for light rays will now have index $2^{M-j}$ (this is the eccentricity of tubes dual to our new plates):

$$
\begin{gathered}
\left\|\sum_{\mathbf{N}} \mathcal{F}^{-1}\left[\widetilde{\Phi}_{M, \mathbf{N}} \varphi_{j}\right] * f\right\|_{L^{p_{0}}}=\left\|\sum_{\mathbf{N}} \mathcal{F}^{-1}\left[\widetilde{\Phi}_{M, \mathbf{N}} \varphi_{j}\right] * \widetilde{f}_{\mathbf{N}}\right\|_{L^{p_{0}}} \\
\lesssim 2^{j \lambda^{*}\left(p_{0}\right)}\left\|\left(\sum_{\mathbf{N}, \nu}\left|\mathcal{F}^{-1}\left[\widetilde{\Phi}_{M, \mathbf{N}} \varphi_{j}\right] * \widetilde{f}_{\mathbf{N}}\right|^{2}\right)^{\frac{1}{2}}\right\|_{L^{p_{0}}} \\
\left\|\left(\sum_{\mathbf{N}}\left|\mathcal{F}^{-1}\left[\widetilde{\Phi}_{M, \mathbf{N}} \varphi_{j}\right] * \widetilde{f}_{\mathbf{N}}\right|^{2}\right)^{\frac{1}{2}}\right\|_{L^{p_{0}}}^{2} \\
=\left(\left.\left.\int\left|\sum_{\mathbf{N}}\right| \mathcal{F}^{-1}\left[\widetilde{\Phi}_{M, \mathbf{N}} \varphi_{j}\right] * \widetilde{f}_{\mathbf{N}}\right|^{2}\right|^{\frac{p_{0}}{2}}\right)^{\frac{2}{p_{0}}} \\
=\sup _{\|\omega\|} \int\left(\sum_{\left(\frac{p_{0}}{2}\right)^{\prime}} \leq 1\right. \\
\left.\left|\mathcal{F}^{-1}\left[\widetilde{\Phi}_{M, \mathbf{N}} \varphi_{j}\right] * \widetilde{f}_{\mathbf{N}}\right|^{2}\right) \omega .
\end{gathered}
$$


Let $\|\omega\|_{\left(\frac{p_{0}}{2}\right)^{\prime}} \leq 1$. Then

$$
\begin{gathered}
\sup _{\|\omega\|_{\left(\frac{p_{0}}{2}\right)^{\prime} \leq 1}} \int\left(\sum_{\mathbf{N}}\left|\mathcal{F}^{-1}\left[\widetilde{\Phi}_{M, \mathbf{N}} \varphi_{j}\right] * \widetilde{f}_{\mathbf{N}}\right|^{2}\right) \omega \\
\lesssim \sum_{m=0}^{\infty} 2^{-m} \int\left(\sum_{\mathbf{N}} \frac{1}{\left|\widetilde{R}_{m}^{\mathbf{N}}\right|} \chi_{\widetilde{R}_{m}^{\mathbf{N}}} * \widetilde{f}_{\mathbf{N}}\right) \omega \\
\int\left(\sum_{\mathbf{N}} \frac{1}{\left|\widetilde{R}_{m}^{\mathbf{N}}\right|} \chi_{\widetilde{R}_{m}^{\mathbf{N}}} * \widetilde{f}_{\mathbf{N}}\right) \omega \leq \int \sum_{\mathbf{N}}\left(\int \frac{1}{\left|\widetilde{R}_{m}^{\mathbf{N}}\right|} \chi_{\widetilde{R}_{m}^{\mathbf{N}}}(y) d y\right)
\end{gathered}
$$

$$
\begin{aligned}
& \left(\int \frac{1}{\left|\widetilde{R}_{m}^{\mathbf{N}}\right|} \chi_{\widetilde{R}_{m}^{\mathbf{N}}}(x-y)\left|\widetilde{f}_{\mathbf{N}}(y)\right|^{2} d y\right) \omega(x) d x \\
\leq & \int \sum_{\mathbf{N}}\left|\widetilde{f}_{\mathbf{N}}(y)\right|^{2} \int \frac{1}{\left|\widetilde{R}_{m}^{\mathbf{N}}\right|} \chi_{\widetilde{R}_{m}^{\mathbf{N}}}(x-y) \omega(x) d x d y
\end{aligned}
$$$$
\leq \int \sum_{\mathbf{N}}\left|\widetilde{f}_{\mathbf{N}}(y)\right|^{2} M_{2^{j-M}} \omega(y) d y
$$

$$
\left\|\sum_{\mathbf{N} \in S_{M}} \mathcal{F}^{-1}\left[\widetilde{\Phi}_{M, \mathbf{N}} \varphi_{j}\right] * f\right\|_{L^{p_{0}}} \lesssim 2^{M \lambda^{*}\left(p_{0}\right)} 2^{\frac{(M-j) a}{2}}\|f\|_{L^{p_{0}}} .
$$

As above, interpolation with the assumed bound at $p=p_{c}$ yields (2.37).

2.3.3. Preparation of the multiplier. We now apply Propositions 2.3 and 2.5 to $m_{j k}$. First we decompose $m_{j k}$ into pieces to which the propositions can be applied. Let

$$
\begin{aligned}
\mu_{j k}(\xi) & =m_{j k}(\xi)-\varphi\left(2^{j}\left(1-\left|\xi^{\prime}\right|\right)\right) \varphi\left(2^{k}\left(1-\left|\xi_{d+1}\right|\right)\right)\left(1-\left|\xi^{\prime}\right|\right)^{\lambda} \\
& = \begin{cases}\varphi\left(2^{j}\left(1-\left|\xi^{\prime}\right|\right)\right) \varphi\left(2^{k}\left(1-\left|\xi_{d+1}\right|\right)\right)\left[\left(1-\left|\xi_{d+1}\right|\right)^{\lambda}-\left(1-\left|\xi^{\prime}\right|\right)^{\lambda}\right] \\
\text { if }\left|\xi_{d+1}\right| \geq\left|\xi^{\prime}\right|, \\
0 & \text { if }\left|\xi_{d+1}\right| \leq\left|\xi^{\prime}\right| .\end{cases}
\end{aligned}
$$

Referring back to the proof of Lemma 2.1 we see that the multiplier we are subtracting,

$$
\varphi\left(2^{j}\left(1-\left|\xi^{\prime}\right|\right)\right) \varphi\left(2^{k}\left(1-\left|\xi_{d+1}\right|\right)\right)\left(1-\left|\xi^{\prime}\right|\right)^{\lambda}
$$


is nearly the same as the multipliers considered in Case (i). The only difference is that here $|k-j| \leq 1$, whereas in Case (i) $j \geq k+2$. The same proof applies here, so

$$
\left\|\varphi\left(2^{j}\left(1-\left|(\cdot)^{\prime}\right|\right)\right) \varphi\left(2^{k}\left(1-\left|(\cdot)_{d+1}\right|\right)\right)\left(1-\left|(\cdot)^{\prime}\right|\right)^{\lambda}\right\|_{M^{p}} \lesssim 2^{j\left(-\lambda+\lambda^{*}(p)+\epsilon\right)} .
$$

We can sum these terms for $|k-j| \leq 1, j \geq 2$, as long as $\lambda>\lambda^{*}(p)+\epsilon$.

Case (iii) is now reduced to finding a bound for $\left\|\mu_{j k}\right\|_{M_{p}}$.

Fix $j, k$ such that $|j-k| \leq 1$ and $j, k \neq 1$. Decompose the support of $\mu_{j k}$ in the following manner. Let $\phi \in \mathcal{C}_{0}^{\infty}(\mathbf{R})$ be a function such that $\phi \equiv 1$ on $\left[\frac{1}{4}, 4\right]$ and $\operatorname{supp} \phi \subset\left[\frac{1}{8}, 8\right]$. Let $\widetilde{\varphi}(t)=\varphi(t) t$ and $\widetilde{\phi}(t)=\phi(t) t^{\lambda-1}$. Then

$$
\begin{aligned}
\left(1-\left|\xi_{d+1}\right|\right)^{\lambda}-\left(1-\left|\xi^{\prime}\right|\right)^{\lambda} & \int_{0}^{1}-\lambda\left[1-\left(t\left|\xi_{d+1}\right|+(1-t)\left|\xi^{\prime}\right|\right)\right]^{\lambda-1}\left[\left|\xi_{d+1}\right|-\left|\xi^{\prime}\right|\right] d t \\
= & -\lambda\left|\xi_{d+1}\right|\left|1-\frac{\left|\xi^{\prime}\right|}{\left|\xi_{d+1}\right|}\right| \int_{0}^{1}\left[1-\left(t\left|\xi_{d+1}\right|+(1-t)\left|\xi^{\prime}\right|\right)\right]^{\lambda-1} d t \\
\varphi & \left(2^{M} h(\xi)\right) \mu_{j k}(\xi) \\
= & \varphi\left(2^{M} h(\xi)\right) \varphi\left(2^{j}\left(1-\left|\xi^{\prime}\right|\right)\right) \\
& \cdot \quad \varphi\left(2^{k}\left(1-\left|\xi_{d+1}\right|\right)\right)\left[\left(1-\left|\xi_{d+1}\right|\right)^{\lambda}-\left(1-\left|\xi^{\prime}\right|\right)^{\lambda}\right] \\
= & \varphi\left(2^{M} h(\xi)\right) \varphi\left(2^{j}\left(1-\left|\xi^{\prime}\right|\right)\right) \varphi\left(2^{k}\left(1-\left|\xi_{d+1}\right|\right)\right) \\
& \cdot \quad \int_{0}^{1}\left(\phi\left(2^{j}\left[1-\left(t\left|\xi_{d+1}\right|+(1-t)\left|\xi^{\prime}\right|\right)\right]\right)(-\lambda)\left|\xi_{d+1}\right|\right. \\
= & \left.\quad\left|1-\frac{\left|\xi^{\prime}\right|}{\left|\xi_{d+1}\right|}\right|\left[1-\left(t\left|\xi_{d+1}\right|+(1-t)\left|\xi^{\prime}\right|\right)\right]^{\lambda-1}\right) d t \\
& \cdot \quad \varphi\left(2^{k}\left(1-\left|\xi_{d+1}\right|\right)\right) \int_{0}^{-M} \widetilde{\phi}\left(1-\left(t\left|\xi_{d+1}\right|+(1-t)\left|\xi^{\prime}\right|\right)\right) d t \\
= & (-\lambda)\left|\xi_{d+1}\right| 2^{-M} 2^{-j(\lambda-1)} \widetilde{\varphi}\left(2^{M}(h(\xi))\right) \int_{0}^{1} \phi_{j, t}(\xi) d t \\
\phi_{j, t}(\xi)= & \varphi\left(2^{j}\left(1-\left|\xi^{\prime}\right|\right)\right) \varphi\left(2^{k}\left(1-\left|\xi_{d+1}\right|\right)\right) \widetilde{\phi}\left(1-\left(t\left|\xi_{d+1}\right|+(1-t)\left|\xi^{\prime}\right|\right)\right)
\end{aligned}
$$

Since $|k-j| \leq 1, \phi_{j, t}$ as defined in (2.75) clearly satisfies the conditions for Propositions 2.3 and 2.5, with all derivative bounds uniform for $t \in[0,1]$. The multiplier $-\lambda\left|\xi_{d+1}\right|$, when smoothly cut off outside of the cylinder, represents a nice bounded multiplier operator so we can disregard it. We can now complete the calculation.

Prior to proceeding, however, we note that with the relationship to the cone multiplier shown above, we can verify the following result of Luers [11, mentioned earlier in the introduction.

Proposition 2.11. If $d \geq 3$ and $\lambda^{*}(p) \geq 1$, then $S^{\lambda}$ is unbounded for all $\lambda$.

Set $k=3$. Choose $\varphi \in \mathcal{C}_{0}^{\infty}(\mathbf{R})$ so that the closure of the support of $\varphi$ is contained in the support of $\varphi(\bar{\varphi}$ as chosen just prior to line $(2.5))$, and so that $\underline{\varphi}(t) \equiv 1$ for 
$t \in\left[\frac{7}{8}, \frac{5}{4}\right]$ ( $\varphi$ was chosen to be non-zero on the interval). Define a new operator $C$ by

$$
\widehat{C f}(\xi)=\left(1-\frac{\left|\xi^{\prime}\right|}{\left|\xi_{d+1}\right|}\right)_{+}^{1} \underline{\varphi}\left(2^{3}\left(1-\left|\xi_{d+1}\right|\right)\right) \hat{f}(\xi) .
$$

This operator clearly has equivalent boundedness properties as the cone multiplier operator $C^{\lambda}$ defined in the introduction, with $\lambda=1$. We define another new operator $\mathcal{E}$ as follows:

$$
\begin{aligned}
\varepsilon(\xi)= & \varphi\left(2^{3}\left(1-\left|\xi^{\prime}\right|\right)\right) \varphi\left(2^{3}\left(1-\left|\xi_{d+1}\right|\right)\right) \\
& \cdot \quad \int_{0}^{1} \varphi\left(2^{3}\left[1-\left(t\left|\xi_{d+1}\right|+(1-t)\left|\xi^{\prime}\right|\right)\right]\right)(-\lambda)\left|\xi_{d+1}\right| \\
& \cdot\left[1-\left(t\left|\xi_{d+1}\right|+(1-t)\left|\xi^{\prime}\right|\right)\right]^{\lambda-1} d t \\
\widehat{\mathcal{E f}}(\xi)= & \underline{\varphi}\left(2^{3}\left(1-\left|\xi^{\prime}\right|\right)\right) \underline{\varphi}\left(2^{3}\left(1-\left|\xi_{d+1}\right|\right)\right)(\varepsilon(\xi))^{-1} .
\end{aligned}
$$

Note that $\mathcal{E}$ has as its multiplier a nice Schwartz function, so $\mathcal{E}$ is trivially bounded for all $p$. Comparing the definition of $\mathcal{E}$ with (2.72), one sees that the composed operator $\mathcal{F}^{-1}\left[\mu_{3,3} \widehat{\mathcal{E f}}\right]$ differs from $C$ only by a simple operator, which we will denote $\mathcal{I}$ :

$$
\widehat{\mathcal{I f}}(\xi)=\widehat{C f}(\xi)-\mu_{3,3} \widehat{\mathcal{E f}}(\xi) .
$$

$\mathcal{I}$ has for its multiplier the same multiplier as $C$, except bounded away from the light cone (which is the only place where $C$ is bad). One sees that $\mathcal{I}$ is also trivially a bounded operator for all $p$.

It is clear from the work done thus far that the boundedness of $S^{\lambda}$ implies the boundedness of the operator $\mathcal{F}^{-1}\left[m_{3,3} \widehat{f}\right]$ and hence also the boundedness of $\mathcal{F}^{-1}\left[\mu_{3,3} \widehat{f}\right]$. Therefore, it would also imply the boundedness of $C$. However, the necessary condition for the cone multiplier operator (see line (2.4) ) is well known, and $C$ can only be bounded for $\lambda^{*}(p)<1$. Thus we have the necessary condition $\lambda^{*}(p)<1$ for boundedness of $S^{\lambda}$, independent of $\lambda$.

2.3.4. Applying Proposition 2.3. To establish the bound (2.15), we relate our multiplier to spherical Bochner-Riesz means in $\mathbf{R}^{d}$.

Lemma 2.12. Suppose $\eta \in \mathcal{C}_{0}^{\infty}\left(\mathbf{R}^{d}\right)$ is supported in a $\delta$-neighborhood of the set $\left\{\xi^{\prime} \in \mathbf{R}^{d}:\left|\xi^{\prime}\right|=t\right\}, \frac{1}{2}<t<1$. Suppose $\eta$ satisfies the condition $\left|\nabla^{n} \eta\right|<C$ for all $0 \leq n \leq N$, where $N$ is a large constant. Assume that for all $\eta$ satisfying these conditions, we have

$$
\|\eta\|_{M^{p_{0}}} \leq C^{\prime} \delta^{-\lambda^{*}\left(p_{0}\right)+\epsilon}
$$

where $C^{\prime}$ depends only on $C, p_{0}, N$ and $d$ (independent of $t, \eta$ ). Then

$$
\left\|\varphi\left(2^{M} h(\cdot)\right) \chi\left(2^{M(1+\epsilon)}\left((\cdot)_{d+1}-\frac{\nu}{2^{M(1+\epsilon)}}\right)\right) \varphi_{j}\right\|_{M^{p_{0}}} \leq C_{1} C_{\varphi} 2^{M\left(\lambda^{*}\left(p_{0}\right)+\epsilon\right)} .
$$

Proof. We proceed by taking $\varphi\left(2^{M} h(\xi)\right)$ and expanding it in a Taylor series in the $\xi_{d+1}$ variable around $\frac{\nu}{2^{M(1+\epsilon)}}$. Denote $\chi_{\nu}\left(\xi_{d+1}\right)=\chi\left(2^{M(1+\epsilon)}\left(\xi_{d+1}-\frac{\nu}{2^{M(1+\epsilon)}}\right)\right)$. 
Then

$$
\begin{aligned}
& \varphi\left(2^{M} h(\xi)\right) \chi_{\nu}\left(\xi_{d+1}\right) \varphi_{j}(\xi) \\
& =\left.\chi_{\nu}\left(\xi_{d+1}\right) \varphi_{j}(\xi) \sum_{n=0}^{\infty} \frac{1}{n !} \frac{\partial^{n}}{\partial \xi_{d+1}^{n}}\left[\varphi\left(2^{M} h(\xi)\right)\right]\right|_{\xi_{d+1}=\frac{\nu}{2^{M(1+\epsilon)}}}\left(\xi_{d+1}-\frac{\nu}{2^{M(1+\epsilon)}}\right)^{n} .
\end{aligned}
$$

Each derivative in the series loses $2^{M}$ but we gain $2^{-M(1+\epsilon)}$ due to the support of $\chi_{\nu}\left(\xi_{d+1}\right)$. Therefore, we need only consider the first $N$ terms, which all clearly satisfy the $\eta$ condition. The $\varphi_{j}$ term can be absorbed into $\eta$, and the $\chi$ term represents a nice bounded operator (its kernel is $L^{1}$ independent of $M$ ).

With this lemma, we now have (2.15) for $p_{0}$ where the Bochner-Riesz conjecture is known in $\mathbf{R}^{d}$. Specifically, we have (2.15) for all $p_{0}$ when $d=2$ (see [3], 4]) and for $d \geq 3$ we have it for $p_{0}>2+\frac{4}{d}$ due to Lee [10].

For the bound (2.16), we use a trivial bound in $\mathbf{R}^{2} \times \mathbf{R}$ and $\mathbf{R}^{3} \times \mathbf{R}$, and the bound of Łaba and Wolff [9] for $d \geq 4$.

For $d=2$ and $d=3$, we note that the same simple integration by parts argument used to show Lemma 2.7 also implies the following:

$$
\left\|\mathcal{F}^{-1}\left[\Phi_{M, \mathbf{N}} \varphi_{j}\right]\right\|_{L^{1}} \lesssim 1 .
$$

The $L^{1}$ norm of the kernel bounds the operator norm (for all $p$ ) so we just add up the pieces:

$$
\begin{aligned}
\left\|\varphi\left(2^{M} h(\xi)\right) \varphi_{j}\right\|_{M^{\infty}} & \lesssim\left\|\sum_{\mathbf{N} \in S_{M}} \Phi_{M, \mathbf{N}} \varphi_{j}\right\|_{M^{\infty}} \\
& \lesssim \sum_{\mathbf{N} \in S_{M}} 1 \\
& \approx 2^{\left(\frac{M}{2}\right)(d-1)} \\
& =2^{M \lambda^{*}(\infty)}
\end{aligned}
$$

For $d \geq 4$, we use the following result of taba and Wolff [9]. Let $\Gamma_{N}(C)$ denote the $C$-neighborhood of the cone segment $\left\{\xi: 2^{-C} N \leq|\xi| \leq 2^{C} N\right\}$. For fixed large $N$, we take an open covering of $S^{d-1}$ by caps $\Theta$ of diameter about $N^{-\frac{1}{2}}$, and let $\Xi_{\Theta}$ be functions whose Fourier transforms restricted to $\Gamma_{N}(C)$ form a partition of unity, subordinate to $\{\Theta\}$ in the natural way. If the support of $\widehat{f}$ is contained in $\Gamma_{N}(1)$, we define

$$
\begin{aligned}
\|f\|_{p, m i c} & =\left(\sum_{\Theta}\left\|\Xi_{\Theta} * f\right\|_{p}^{p}\right)^{\frac{1}{p}} \quad \text { for } 2 \leq p<\infty, \text { and } \\
\|f\|_{\infty, m i c} & =\sup _{\Theta}\left\|\Xi_{\Theta} * f\right\|_{\infty} .
\end{aligned}
$$

Theorem 2.13 (Laba and Wolff). The following estimate holds in $\mathbf{R}^{d} \times \mathbf{R}$ if $d \geq 3$, $p>p_{d} \stackrel{\text { def }}{=} \min \left(2+\frac{8}{d-3}, 2+\frac{32}{3 d-7}\right)$ and $\widehat{f}$ is supported in $\Gamma_{N}(1)$ :

$$
\forall \epsilon \exists C_{\epsilon}:\|f\|_{p} \leq C_{\epsilon} N^{\epsilon} N^{d\left(\frac{1}{2}-\frac{1}{p}\right)-\frac{1}{2}}\|f\|_{p, m i c} .
$$


Our terms $\Phi_{M, \mathbf{N}}$, after scaling by $2^{M}$, are supported on the cone $\Gamma_{2^{M}}(1)$. In the following $p_{c}>p_{d}$ :

$$
\begin{aligned}
\left\|\mathcal{F}^{-1}\left[\varphi\left(2^{M} h(\xi)\right) \varphi_{j}\right] * f\right\|_{p_{c}} & \\
& \left.\lesssim 2^{M \epsilon} 2^{M \lambda^{*}\left(p_{c}\right)}\left(\sum_{\mathbf{N} \in S_{M}} \| \mathcal{F}^{-1}\left[\Phi_{M, \mathbf{N}} \varphi_{j}\right] * f\right] \|_{p_{c}}^{p_{c}}\right)^{\frac{1}{p_{c}}} \\
& \lesssim 2^{M\left(\lambda^{*}\left(p_{c}\right)+\epsilon\right)}\|f\|_{p_{c}} .
\end{aligned}
$$

Above, (2.86) follows from (2.85) (2.87) can be obtained easily by checking the $L^{2}$ and $L^{\infty}$ cases (using the bound from line (2.83)), then interpolating.

Line (2.87) and Lemma 2.12 provide the bounds one needs for Proposition 2.3 . We apply Proposition 2.3 in line (2.89). For $p_{0} \leq p \leq p_{c}$, we have the following:

$$
\begin{aligned}
\sum_{|j-k| \leq 1}\left\|\mu_{j k}\right\|_{M^{p}}=\sum_{|j-k| \leq 1}\left\|\sum_{M=j-6}^{\infty} \varphi\left(2^{M} h(\xi)\right) \mu_{j k}\right\|_{M^{p}} \\
\quad \lesssim \sum_{j=1}^{\infty}\left(\sum_{M=j-6}^{\infty} 2^{-M_{2}-j(\lambda-1)} 2^{(M-j)\left(\frac{\frac{1}{p}-\frac{1}{p_{c}}}{p_{0}}\right)\left(1-\frac{2}{p_{c}}\right)} 2^{M\left(\lambda^{*}(p)+\epsilon\right)}\right) \\
\quad \lesssim \sum_{j=1}^{\infty}\left(2^{-j\left(\lambda-\lambda^{*}(p)-\epsilon\right)}\right) .
\end{aligned}
$$

For the sums to converge as indicated above, we require

$$
\lambda^{*}(p)+\left(\frac{\frac{1}{p}-\frac{1}{p_{c}}}{\frac{1}{p_{0}}-\frac{1}{p_{c}}}\right)\left(1-\frac{2}{p_{0}}\right)+\epsilon<1 .
$$

For specific dimensions, our results are as follows:

- In $\mathbf{R}^{2} \times \mathbf{R}, p_{0}=4$ and $p_{c}=\infty$. We have convergence for $\lambda>\lambda^{*}(p)$, $4 \leq p \leq \infty$.

- In $\mathbf{R}^{3} \times \mathbf{R}, p_{0}=2+\frac{4}{d}=\frac{10}{3}$ and $p_{c}=\infty$. We have convergence for $\lambda>\lambda^{*}(p), \frac{10}{3}<p<\infty$.

- In $\mathbf{R}^{4} \times \mathbf{R}, p_{0}=2+\frac{4}{d}=3$ and $p_{c}=2+\frac{32}{3 d-7}=\frac{42}{5}$. We have convergence for $\lambda>\lambda^{*}(p), 3<p<2+\frac{6}{d-3}-\frac{4}{17}=\frac{132}{17}$.

- In $\mathbf{R}^{d} \times \mathbf{R}$ for $d \geq 5, p_{0}=2+\frac{4}{d}$ and $p_{c}=2+\frac{8}{d-3}$. We have convergence for $\lambda>\lambda^{*}(p), 2+\frac{4}{d}<p<2+\frac{6}{d-3}-\frac{4}{(d-3)(d+1)}$.

Note that $\lambda^{*}\left(2+\frac{6}{d-3}\right)=1$.

2.4. Applying Proposition 2.5. Throughout this section, we will assume $d \geq 4$ since Proposition 2.3 gave the best possible results for $d=2,3$ already. We have (2.34) with $q_{0}=2$ (so $p_{0}=4$ ) and $a=0$ (no loss!) due to Mockenhaupt, Seeger and Sogge [14. Unfortunately, we can only use this result for $d=4$ and $d=5$ since $q_{0}=\left(\frac{p_{0}}{2}\right)^{\prime}$ and we require that $p_{0} \geq \frac{2(d+1)}{d-1}$. For $d \geq 6$, we use a different bound with $q_{0}=\frac{d+1}{2}$ and $a=\frac{1}{d+1}$, which is due to Skarabot [17. This is proved using Bourgain's bush argument; an alternative variation of the bush argument is presented in 3.3. Note that if $p_{0}=\frac{2(d+1)}{d-1}$, then $q_{0}=\left(\frac{p_{0}}{2}\right)^{\prime}=\frac{d+1}{2}$, so Skarabot's result can be used in Proposition 2.5 for any dimension. 
With Skarabot's result and line (2.87), we can apply Proposition 2.5 (we use it in line (2.93) below). For $\frac{2(d+1)}{d-1} \leq p \leq p_{c}$,

$$
\begin{aligned}
& \sum_{|j-k| \leq 1}\left\|\mu_{j k}\right\|_{M^{p}}=\sum_{|j-k| \leq 1}\left\|\sum_{M=j-6}^{\infty} \varphi\left(2^{M} h(\xi)\right) \mu_{j k}\right\|_{M^{p}} \\
& \quad \lesssim \sum_{j=1}^{\infty}\left(\sum_{M=j-6}^{2 j} 2^{-M} 2^{-j(\lambda-1)} 2^{(M-j)\left(\frac{a}{2}\right)\left(\frac{\frac{1}{p}-\frac{1}{p_{c}}}{p_{0}}-\frac{1}{p_{c}}\right.}\right) 2^{M\left(\lambda^{*}(p)+\epsilon\right)} \\
& \left.\quad+\sum_{M=2 j}^{\infty} 2^{-M} 2^{-j(\lambda-1)} 2^{\left.\left(\frac{M}{2}-j\right)\left(\frac{1}{2}-\frac{1}{p_{0}}\right)+M\left(\frac{a}{4}\right)\right)\left(\frac{\frac{1}{p}-\frac{1}{p_{c}}}{\frac{1}{p_{0}}-\frac{-1}{p_{c}}}\right)} 2^{M\left(\lambda^{*}(p)+\epsilon\right)}\right) \\
& \quad \lesssim \sum_{j=1}^{\infty}\left(2^{-j\left(\lambda-\lambda^{*}(p)-\epsilon\right)}\right)
\end{aligned}
$$

provided we have the following two inequalities:

$$
\begin{aligned}
\left(\frac{a}{2}\right)\left(\frac{\frac{1}{p}-\frac{1}{p_{c}}}{\frac{1}{p_{0}}-\frac{1}{p_{c}}}\right)+\lambda^{*}(p)+\epsilon & <1, \\
\left(\frac{1}{2}\left(\frac{1}{2}-\frac{1}{p_{0}}\right)+\frac{a}{4}\right)\left(\frac{\frac{1}{p}-\frac{1}{p_{c}}}{\frac{1}{p_{0}}-\frac{1}{p_{c}}}\right)+\lambda^{*}(p) & <1 .
\end{aligned}
$$

Note that the second inequality implies the first for $\frac{a}{4}<\frac{1}{2}\left(\frac{1}{2}-\frac{1}{p_{0}}\right)$. This is true both for $a=\frac{1}{d+1}, p_{0}=\frac{2(d+1)}{d-1}$ and for $a=0, p_{0}=4$. It is interesting to note that (2.96) comes from the case where $M$ is much larger than $j$. When $M \gg j$, we are especially close to the light cone where the multiplier $m_{\lambda}$ is not smooth. This observation captures the fact that for $\lambda^{*}(p)$ near 1, the quality of our result depends directly on our knowledge of the cone multiplier (and truncated cone multipliers).

After working out the algebra, Proposition 2.5 provides the following results. It turns out that even though we have a maximal function bound with $a=0$ for $d=4,5$, it is still more efficient to use the $a=\frac{1}{d+1}$ bound. This is due to the fact that $\frac{2(d+1)}{d-1}<4$. In this case, the gain from interpolating with a smaller $p_{0}$ exceeds the gain from having a better value for $a$.

- In $\mathbf{R}^{4} \times \mathbf{R}, p_{c}=2+\frac{32}{3 d-7}=\frac{42}{5}$. With $p_{0}=\frac{2(d+1)}{d-1}$ and $a=\frac{1}{d+1}$, we obtain convergence for $\lambda>\lambda^{*}(p), \frac{2(d+1)}{d-1}<p<2+\frac{6}{d-3}-\frac{6}{61}=\frac{482}{61}$. With $a=0$ and $p_{0}=4$, we obtain convergence for $4<p<2+\frac{6}{d-3}-\frac{2}{17}=\frac{134}{17}$.

- In $\mathbf{R}^{5} \times \mathbf{R}, p_{c}=2+\frac{8}{d-3}=6$. With $p_{0}=\frac{2(d+1)}{d-1}$ and $a=\frac{1}{d+1}$, we obtain convergence for $\lambda>\lambda^{*}(p), \frac{2(d+1)}{d-1}<p<2+\frac{6}{d-3}-\frac{1}{7}=\frac{34}{7}$. With $a=0$ and $p_{0}=4$, we obtain convergence for $4<p<2+\frac{6}{d-3}-\frac{1}{3}=\frac{14}{3}$.

- In $\mathbf{R}^{d} \times \mathbf{R}$ for $d \geq 6, p_{0}=\frac{2(d+1)}{d-1}, a=\frac{1}{d+1}$ and $p_{c}=2+\frac{8}{d-3}$. We have convergence for $\lambda>\lambda^{*}(p), \frac{2(d+1)}{d-1}<p<\frac{8 d^{2}+2 d-6}{4 d^{2}-11 d-3}=2+\frac{6}{d-3}-\frac{6}{(4 d+1)(d-3)}$. 
Note that for $d$ large, we were off of the best value of $2+\frac{6}{d-3}$ by $\sim \frac{4}{d^{2}}$ using Proposition 2.3. Now we lose only $\sim \frac{3}{2 d^{2}}$, which is a significant improvement.

Together, the two sets of ranges for $p$ from Propositions 2.3 and 2.5 provide the ranges stated in 1

2.5. Case (iv) $(k, j) \in\{(1,1),(1,2),(2,1)\}$, the center.

\section{Lemma 2.14.}

$$
\left\|\mathcal{F}^{-1}\left[m_{11}+m_{12}+m_{21}\right] * f\right\|_{L^{p}} \lesssim\|f\|_{L^{p}}
$$

for all $f \in L^{p}\left(\mathbf{R}^{d} \times \mathbf{R}\right), 1<p<\infty$ and $\lambda>0$.

Proof. In this region we use the following theorem of Seeger [15] to extend our result to the origin. The theorem applies with $\varphi$ defined as in the rest of this paper.

Theorem 2.15 (Seeger). Suppose that $m$ is a bounded function which satisfies for some $p, 1<p<\infty, \epsilon>0$,

$$
\begin{aligned}
\sup _{t>0}\|\varphi(|\cdot|) m(t \cdot)\|_{M^{p}} & \leq A, \\
\sup _{t>0} \int_{|x| \geq w}\left|\mathcal{F}^{-1}[\varphi(|\cdot|) m(t \cdot)](x)\right| d x & \leq B(1+w)^{-\epsilon} .
\end{aligned}
$$

Then

$$
\|m\|_{M^{p}} \lesssim A\left[\log \left(2+\frac{B}{A}\right)\right]^{\frac{1}{p}-\frac{1}{2}}
$$

We begin by decomposing the multiplier in this region similarly to the way we did in (2.3). Note we work with $\mu_{11}$ and the other two multipliers are similar:

$$
\mu_{11}(\xi)=\sum_{M=0}^{\infty} \varphi\left(2^{M} h(\xi)\right)\left[\left(1-\left|\xi_{d+1}\right|\right)^{\lambda}-\left(1-\left|\xi^{\prime}\right|\right)^{\lambda}\right] \varphi_{1}\left(\left|\xi^{\prime}\right|\right) \varphi_{1}\left(\left|\xi_{d+1}\right|\right)
$$

Denote the terms in the sum $\mu_{M}(\xi)$. By analyzing the multiplier $\varphi(|\cdot|) \mu_{M}(t \cdot)$, we can obtain boundedness results for $\mu_{11}$. First we note that due to the support of $\mu_{M}, \varphi(|\cdot|) \mu_{M}(t \cdot)$ is only non-zero if $t<2$. By analyzing derivatives of the multiplier and using the fact that $t$ is bounded, we see that

$$
\sup _{t>0}\left\|\varphi(|\cdot|) \mu_{M}(t \cdot)\right\|_{L_{\alpha}^{2}} \leq C_{\alpha} 2^{-M} \quad \text { independent of } t .
$$

We use this bound for the condition (2.98) in Theorem 2.15.

We obtain a bound of the form on (2.99) by doing integration by parts $d+2$ times. Note that in the integration by parts we lose at most $2^{M}$ on each derivative since that is the loss in the multiplier's worst direction. We begin by defining the 
set $\Omega_{j}^{\omega}$ :

$$
\Omega_{j}^{\omega}=\left\{\xi \in \mathbf{R}^{d} \times \mathbf{R},|\xi|>\omega \text { and }\left|\xi_{j}\right|=\max _{k=1, \ldots, d+1}\left|\xi_{k}\right|\right\}
$$

$$
\sup _{t>0} \int_{|x|>\omega}\left|\mathcal{F}^{-1}\left[\varphi(|\cdot|) \mu_{M}(t \cdot)\right](x)\right| d x \lesssim \sup _{t>0} \int_{|x|>\omega}\left|\int e^{i x \cdot \xi} \varphi(|\xi|) \mu_{M}(t \xi) d \xi\right| d x
$$

$$
\begin{aligned}
& \lesssim \sup _{t>0} \sum_{j=1}^{d+1} \int_{\Omega_{j}^{\omega}}\left|\int \frac{e^{i x \cdot \xi}}{\left|x_{j}\right|^{d+2}} \frac{\partial^{d+1}}{\partial \xi_{j}^{d+1}}\left(\varphi(|\cdot|) \mu_{M}(t \cdot)\right)(\xi) d \xi\right| d x \\
& \lesssim \sup _{t>0} \sum_{j=1}^{d+1} \int_{\Omega_{j}^{\omega}} \int \frac{2^{M(d+2)}}{\left|x_{j}\right|^{d+2}} \chi_{\operatorname{supp}\left(\varphi(|\cdot|) \mu_{M}(t \cdot)\right)}(\xi) d \xi d x \\
& \lesssim \sup _{t>0} \sum_{j=1}^{d+1} \int_{\Omega_{j}^{\omega}} \frac{2^{M(d+1)}}{\left|x_{j}\right|^{d+2}} d x \\
& \lesssim 2^{(d+1) M}(1+\omega)^{-\epsilon} \quad \text { independent of } t .
\end{aligned}
$$

We then obtain a good bound on each $\mu_{M}$ and can sum in $M$,

$$
\begin{aligned}
\left\|\mu_{M}\right\|_{M^{p}} & \lesssim 2^{-M}\left[\log \left(2+\frac{2^{(d+1) M}}{2^{-M}}\right)\right]^{\left|\frac{1}{p}-\frac{1}{2}\right|} \\
& \lesssim M^{\left|\frac{1}{p}-\frac{1}{2}\right|} 2^{-M} \\
\Rightarrow\left\|\mu_{11}\right\|_{M^{p}} & \lesssim \sum_{M=0}^{\infty} M^{\left|\frac{1}{p}-\frac{1}{2}\right|} 2^{-M} \\
& <\infty .
\end{aligned}
$$

This bound on $\left\|\mu_{11}\right\|_{M^{p}}$ applies for $1<p<\infty$ and for all $\lambda>0$.

Combining the results of the four cases completes the proofs for Theorems 1.1. $1.2,1.3$ and 1.4, It remains only to verify the lemmas which we have stated.

\section{Proofs of the Lemmas}

Here we present proofs for Lemmas 2.6, 2.7 and 2.10 and the Nikodym maximal function bound used in 2.4 .

3.1. Proof of Lemma 2.6. Recall that $\chi_{\mathbf{N}, \nu}=\widetilde{\chi}_{Q_{\mathbf{N}}}\left(\xi^{\prime}\right) \widetilde{\chi}_{\nu}\left(\xi_{d+1}\right)$. For generality, denote the radius of the cube where $\chi_{\mathbf{N}, \nu}$ is supported by $\delta$ and let $R=\frac{1}{\delta}\left(\delta=2^{-\frac{M}{2}}\right.$ in Lemma 2.6] and $\delta=2^{-j}$ in Lemma 2.9). $\mathcal{Q}$ was defined to be the cubes in the sum which intersected the support of $\varphi\left(2^{M} h(\cdot)\right) \varphi_{j}$. The union of these cubes will form a $\delta$-neighborhood of this support, which is in the shape of a truncated cone. Also note that for a fixed $\nu$ (so $\left|\xi_{d+1}\right| \sim \frac{\nu}{2^{\frac{M M}{C_{h}}}}$ ), the union of the cubes corresponding to $\nu$ will form a "ring", a $\delta$-neighborhood of a lower dimensional sphere $S^{d-1}$ with radius between $\frac{1}{2}$ and 1 depending linearly on $\nu$.

We follow an argument which is attributed to Stein and can be found in [7]. 
Proof. To show (2.38), it is sufficient to show the following dual inequality for $p \leq \frac{2(d+1)}{d+3}$ :

$$
\left\|\left(\sum\left|\mathcal{F}^{-1}\left[\chi_{\mathbf{N}, \nu}\right] * f\right|^{2}\right)^{\frac{1}{2}}\right\|_{p} \lesssim(\operatorname{card}(\mathcal{V}))^{\left(\frac{1}{2}-\frac{1}{p}\right)} R^{\lambda^{*}\left(p^{\prime}\right)+\epsilon}\|f\|_{p}
$$

Note that we have a bound on the kernels,

$$
\left|\mathcal{F}^{-1}\left[\chi_{\mathbf{N}, \nu}\right](x-y)\right| \leq C_{N} \frac{R^{-(d+1)}}{\left(1+\frac{|x-y|}{R}\right)^{N}}
$$

We may assume that $\mathcal{F}^{-1}\left[\chi_{\mathbf{N}, \nu}\right] * f$ is supported in a cube $\widetilde{Q}=\widetilde{Q}^{\prime} \times \widetilde{Q}_{d+1}$ in $\mathbf{R}^{d} \times \mathbf{R}$ of radius $R^{1+\epsilon}$. Outside of the cube we can use the above bound to gain an arbitrary power of $R$.

In the following, let $f_{\nu}=\mathcal{F}^{-1}\left[\widetilde{\chi}_{\nu}\left(\xi_{d+1}\right) \widehat{f}\right]$. Let $\mathcal{F}_{\xi^{\prime}}=\int_{\mathbf{R}^{d}} e^{i x^{\prime} \cdot \xi^{\prime}} f(x) d x^{\prime}$ denote the partial Fourier transform in the first $d$ variables:

$$
\begin{aligned}
& \left\|\left(\sum\left|\mathcal{F}^{-1}\left[\chi_{\mathbf{N}, \nu}\right] * f\right|^{2}\right)^{\frac{1}{2}}\right\|_{p}^{p}=\int_{\widetilde{Q}}\left(\sum_{\mathbf{N}} \sum_{\nu \in \mathcal{V}}\left|\mathcal{F}^{-1}\left[\chi_{\mathbf{N}, \nu}\right] * f(x)\right|^{2}\right)^{\frac{p}{2}} d x \\
& \quad \lesssim|\widetilde{Q}|^{1-\frac{p}{2}}\left(\int_{\widetilde{Q}} \sum_{\mathbf{N}} \sum_{\nu \in \mathcal{V}}\left|\mathcal{F}^{-1}\left[\chi_{\mathbf{N}, \nu}\right] * f(x)\right|^{2} d x\right)^{\frac{p}{2}} \\
& =|\widetilde{Q}|^{1-\frac{p}{2}}\left(\sum_{\nu \in \mathcal{V}} \int_{\widetilde{Q}_{d+1}} \int_{\mathbf{R}^{d}} \sum_{\mathbf{N}}\left|\widetilde{\chi}_{Q_{\mathbf{N}}}\left(\xi^{\prime}\right) \mathcal{F}_{\xi^{\prime}}\left[f_{\nu}\right]\left(\xi^{\prime}, x_{d+1}\right)\right|^{2} d \xi^{\prime} d x_{d+1}\right)^{\frac{p}{2}} \\
& \quad \lesssim|\widetilde{Q}|^{1-\frac{p}{2}}\left(\sum_{\nu \in \mathcal{V}} R^{-1} \int_{\widetilde{Q}_{d+1}}\left(\int_{\mathbf{R}^{d}}\left|f_{\nu}\right|^{p} d x^{\prime}\right)^{\frac{2}{p}} d x_{d+1}\right)^{\frac{p}{2}} \\
& =R^{p \lambda^{*}(p)+\epsilon} R^{p\left(\frac{1}{p}-\frac{1}{2}\right)}\left(\int_{\widetilde{Q}_{d+1}} \sum_{\nu \in \mathcal{V}}\left(\int_{\mathbf{R}^{d}}\left|f_{\nu}\right|^{p} d x^{\prime}\right)^{\frac{2}{p}} d x_{d+1}\right)^{\frac{p}{2}} .
\end{aligned}
$$

In line (3.2) we are using Hölder's inequality. (3.3) is an application of Plancherel in the first $d$ variables. Then in (3.4) we apply the Tomas-Stein restriction theorem for $\mathbf{R}^{d}$ [18]. The $R^{-1}$ factor comes from the thickness of the annulus in $\mathbf{R}^{d}$. Since $\lambda^{*}\left(p^{\prime}\right)=\lambda^{*}(p)$, it remains to show that

$$
\left(\int_{\widetilde{Q}_{d+1}} \sum_{\nu \in \mathcal{V}}\left(\int_{\mathbf{R}^{d}}\left|f_{\nu}\right|^{p} d x^{\prime}\right)^{\frac{2}{p}} d x_{d+1}\right)^{\frac{1}{2}} \lesssim\left(\frac{\operatorname{card}(\mathcal{V})}{R}\right)^{\left(\frac{1}{p}-\frac{1}{2}\right)} R^{\epsilon}\|f\|_{L^{p}\left(\mathbf{R}^{d} \times \mathbf{R}\right)}
$$

We show this estimate for $p=1$ and $p=2$, then interpolate. For $p=2$, the estimate is trivial by Plancherel's theorem. For $p=1$, first we make the following 
observations of $\chi_{\nu}$ and $f_{\nu}$ :

$$
\begin{aligned}
& \chi_{\nu}\left(\xi_{d+1}\right)=\chi\left(R\left(\xi_{d+1}-\frac{\nu}{R}\right)\right), \\
& \left|\mathcal{F}^{-1}\left[\chi_{\nu}\right]\left(x_{d+1}\right)\right|=\left|\frac{1}{R} \mathcal{F}^{-1}[\chi]\left(\frac{x_{d+1}}{R}\right)\right|, \\
& \left|f_{\nu}(x)\right|=\left|\int_{\mathbf{R}} \mathcal{F}^{-1}\left[\chi_{\nu}\right]\left(x_{d+1}-y_{d+1}\right) f\left(x^{\prime}, y_{d+1}\right) d y_{d+1}\right| \\
& \lesssim\left(\int_{\mathbf{R}}\left|\frac{1}{R} \mathcal{F}^{-1}[\chi]\left(\frac{x_{d+1}-y_{d+1}}{R}\right)\right|^{p^{\prime}} d y_{d+1}\right)^{\frac{1}{p^{\prime}}} \\
& \cdot\left(\int_{\mathbf{R}}\left|f\left(x^{\prime}, y_{d+1}\right)\right|^{p} d y_{d+1}\right)^{\frac{1}{p}} \\
& =\frac{1}{R^{\frac{1}{p}}}\left\|\mathcal{F}^{-1}[\chi]\right\|_{L^{p}(\mathbf{R})}\left(\int_{\mathbf{R}}\left|f\left(x^{\prime}, y_{d+1}\right)\right|^{p} d y_{d+1}\right)^{\frac{1}{p}}, \\
& \left(\int_{\widetilde{Q}_{d+1}} \sum_{\nu \in \mathcal{V}}\left(\int_{\mathbf{R}^{d}}\left|f_{\nu}\right| d x^{\prime}\right)^{2} d x_{d+1}\right)^{\frac{1}{2}} \\
& \lesssim\left(\int_{\widetilde{Q}_{d+1}} \sum_{\nu \in \mathcal{V}}\left(\frac{1}{R} \int_{\mathbf{R}^{d}} \int_{\mathbf{R}}\left|f\left(x^{\prime}, y_{d+1}\right)\right| d y_{d+1} d x^{\prime}\right)^{2} d x_{d+1}\right)^{\frac{1}{2}} \\
& =\frac{1}{R}\left(\int_{\widetilde{Q}_{d+1}} \sum_{\nu \in \mathcal{V}}\|f\|_{L^{1}\left(\mathbf{R}^{d} \times \mathbf{R}\right)}^{2} d x_{d+1}\right)^{\frac{1}{2}} \\
& \lesssim \frac{1}{R}\left(R^{1+\epsilon}(\operatorname{card}(\mathcal{V}))\|f\|_{L^{1}\left(\mathbf{R}^{d} \times \mathbf{R}\right)}^{2}\right)^{\frac{1}{2}} \\
& \lesssim \frac{1}{R^{\frac{1}{2}}} R^{\epsilon}(\operatorname{card}(\mathcal{V}))^{\frac{1}{2}}\|f\|_{L^{1}\left(\mathbf{R}^{d} \times \mathbf{R}\right)} .
\end{aligned}
$$

The estimate (3.6) follows by interpolation. This completes the proof for functions supported in $\widetilde{Q}$. A standard argument, using the gain in (3.1), extends this proof to the general situation.

\subsection{Proofs of Lemmas 2.7 and 2.10 .}

Proof. We begin with the proof of Lemma 2.7. We use integration by parts to bound the kernel $\mathcal{F}^{-1}\left(\Phi_{M, \mathbf{N}, \nu} \varphi_{j}\right)$ in $d+2$ regions, $\Omega_{l}, l=0 \ldots d+1$ :

$$
\begin{gathered}
\Omega_{0}=\left\{x: \max \left\{\left|\frac{\mathbf{u}_{1} \cdot x}{2^{\frac{M}{2}}}\right|, \ldots,\left|\frac{\mathbf{u}_{d-1} \cdot x}{2^{\frac{M}{2}}}\right|,\left|\frac{\mathbf{u}_{d} \cdot x}{2^{M}}\right|,\left|\frac{\mathbf{u}_{d+1} \cdot x}{2^{\frac{M}{2}}}\right|\right\}<2\right\}, \\
\Omega_{l}=\left\{x:\left|\frac{\mathbf{u}_{l} \cdot x}{2^{\frac{M}{2}}}\right|>\frac{1}{2} \max \left\{\left\{\left|\frac{\mathbf{u}_{i} \cdot x}{2^{\frac{M}{2}}}\right|\right\}_{\substack{i=1 \ldots d \\
i \neq l \\
i \neq l}},\left|\frac{\mathbf{u}_{d} \cdot x}{2^{M}}\right|,\left|\frac{\mathbf{u}_{d+1} \cdot x}{2^{\frac{M}{2}}}\right|\right\},\right. \\
\left.x \notin \frac{1}{2} \Omega_{0}\right\} \quad \text { for } 1 \leq l \leq d-1,
\end{gathered}
$$




$$
\begin{aligned}
\Omega_{d} & =\left\{x:\left|\frac{\mathbf{u}_{d} \cdot x}{2^{M}}\right|>\frac{1}{2} \max \left\{\left|\frac{\mathbf{u}_{1} \cdot x}{2^{\frac{M}{2}}}\right|, \ldots,\left|\frac{\mathbf{u}_{d-1} \cdot x}{2^{\frac{M}{2}}}\right|,\left|\frac{\mathbf{u}_{d+1} \cdot x}{2^{\frac{M}{2}}}\right|\right\}, x \notin \frac{1}{2} \Omega_{0}\right\}, \\
\Omega_{d+1} & =\left\{x:\left|\frac{\mathbf{u}_{d+1} \cdot x}{2^{\frac{M}{2}}}\right|>\frac{1}{2} \max \left\{\left|\frac{\mathbf{u}_{1} \cdot x}{2^{\frac{M}{2}}}\right|, \ldots,\left|\frac{\mathbf{u}_{d-1} \cdot x}{2^{\frac{M}{2}}}\right|,\left|\frac{\mathbf{u}_{d} \cdot x}{2^{M}}\right|\right\}, x \notin \frac{1}{2} \Omega_{0}\right\} .
\end{aligned}
$$

Now we apply integration by parts to make our $d+2$ estimates. In the first region, $\Omega_{0}$, we just use the size estimate of $\operatorname{supp}\left(\Phi_{M, \mathbf{N}, \nu} \varphi_{j}\right)$ :

$$
\begin{aligned}
\left|\int e^{i x \cdot \xi} \Phi_{M, \mathbf{N}, \nu}(\xi) \varphi_{j}(\xi) d \xi\right| & \lesssim \int_{\operatorname{supp}\left(\Phi_{M, \mathbf{N}, \nu}\right)} 1 d \xi \\
& \lesssim 2^{-M} 2^{-\frac{M}{2}(d)}
\end{aligned}
$$

On $\Omega_{l}, 1 \leq l \leq d+1$, we integrate by parts $d+2$ times in the dominant direction. For $1 \leq l \leq d-1$, as expected, the derivatives of $\Phi_{M, \mathbf{N}, \nu}$ in the $\mathbf{u}_{l}$-direction each cause a loss of $2^{\frac{M}{2}}$, while derivatives of $\varphi_{j}$ always lose $2^{j}$ :

$$
\begin{aligned}
\left|\int e^{i x \cdot \xi} \Phi_{M, \mathbf{N}, \nu}(\xi) \varphi_{j}(\xi) d \xi\right| & \leq \int \frac{1}{\left(\mathbf{u}_{l} \cdot x\right)^{d+2}}\left(\mathbf{u}_{l} \cdot \nabla\right)^{d+2}\left[\Phi_{M, \mathbf{N}, \nu}(\xi) \varphi_{j}(\xi)\right] d \xi \\
& \lesssim \frac{1}{\left(\mathbf{u}_{l} \cdot x\right)^{d+2}} \int_{\operatorname{supp}\left(\Phi_{M, \mathbf{N}, \nu}\right)} 2^{\frac{M}{2}(d+2)} d \xi \\
& \lesssim \frac{1}{\left(2^{-\frac{M}{2}} \mathbf{u}_{l} \cdot x\right)^{d+2}} 2^{-\frac{M}{2} d} 2^{-M}
\end{aligned}
$$

Similar bounds follow for the $x$ in $\Omega_{d}$ and $\Omega_{d+1}$ :

$$
\begin{aligned}
\left|\int e^{i x \cdot \xi} \Phi_{M, \mathbf{N}, \nu}(\xi) \varphi_{j}(\xi) d \xi\right| & \lesssim \frac{1}{\left(2^{-M} \mathbf{u}_{d} \cdot x\right)^{d+2}} 2^{-\frac{M}{2} d} 2^{-M} \\
\left|\int e^{i x \cdot \xi} \Phi_{M, \mathbf{N}, \nu}(\xi) \varphi_{j}(\xi) d \xi\right| & \lesssim \frac{1}{\left(2^{-\frac{M}{2}} \mathbf{u}_{d+1} \cdot x\right)^{d+2}} 2^{-\frac{M}{2} d} 2^{-M}
\end{aligned}
$$

The lemma follows directly from these estimates.

The proof of Lemma 2.10 is identical. We use the same decompositions and calculations, excepting a few small changes. Derivatives in the directions which lost $2^{\frac{M}{c_{h}}}$ before now lose $2^{j}$, and the size of the support of $\widetilde{\Phi}_{M, \mathbf{N}} \varphi_{j}$ is $2^{-j d} 2^{-M}$ instead of $2^{-\frac{M}{2} d} 2^{-M}$. We also would have to redefine the $\Omega_{l}$ 's by replacing $\frac{\mathbf{u}_{l} \cdot x}{2^{\frac{M}{2}}}$ terms with $\frac{\mathbf{u}_{l} \cdot x}{2^{j}}$.

3.3. Estimate for the Nikodym maximal function for light rays. Here we present a variation of Bourgain's bush argument 11. Skarabot showed this same bound [17] by using the bush argument to show a $L^{\frac{d+1}{2}} \rightarrow L^{d+1}$, then deducing the $L^{p} \rightarrow L^{p}$ bound. We directly derive the $L^{\frac{d+1}{2}} \rightarrow L^{\frac{d+1}{2}}$ bound.

Proof. Since this maximal function uses averages over tubes which are of length 1 and $\delta$ in diameter, it only acts locally. We are able to assume that the input function $f$ and the output $M_{\delta} f$ are supported on a cube of sidelength 4 . We are also able to prove a result in just the first $d$ variables, as we will be able to integrate out the final variable. We verify the following proposition.

Proposition 3.1. Let $2 \leq p \leq d$. Let $E$ be a set contained in the unit cube. Then

$$
\left|\left\{x^{\prime}: M_{\delta} \chi_{E}\left(x^{\prime}, x_{d+1}\right)>\lambda\right\}\right| \lesssim\left(\frac{\delta^{1-\frac{d}{p}-\epsilon} \delta^{-\alpha}|E|^{\frac{1}{p}}}{\lambda}\right)^{p}
$$


where

$$
\alpha= \begin{cases}\frac{1}{2 p} & \text { if } p \leq \frac{d+1}{2}, \\ 1-\frac{d}{2 p} & \text { if } p \geq \frac{d+1}{2} .\end{cases}
$$

By using interpolation on this restricted weak-type bound, we obtain the bound in (2.34) with $a=\frac{1}{d+1}$ and $q_{0}=\frac{d+1}{2}$.

Let $\Omega_{\lambda}=\left\{x^{\prime}: M_{\delta} \chi_{E}\left(x^{\prime}, x_{d+1}\right)>\lambda\right\}$. We begin by taking a maximal $\delta$-separated subset $u_{1}, \ldots, u_{N}$ of $\Omega_{\lambda}$. Note that $\left|\Omega_{\lambda}\right| \leq N \delta^{-d}$. For each $u_{\gamma}$, we choose a tube $R_{\gamma}$, with dimensions $\delta \times \cdots \times \delta \times 1$ and pointing along a light ray, which satisfies

$$
\frac{1}{\left|R_{\gamma}\right|} \int_{R_{\gamma}} \chi_{E}=\frac{\left|E \cap R_{\gamma}\right|}{\left|R_{\gamma}\right|}>\lambda
$$

By using our $N \delta^{-d}$ bound and isolating $|E|$ in (3.16), it suffices to show

$$
|E| \gtrsim N \delta^{2 d-p+\alpha p+\epsilon} \lambda^{p}
$$

We now separate into two cases: When there is no point $\left(x^{\prime}, x_{d+1}\right)$ contained in more than $\mu=\lambda^{1-p} \delta^{p-d-\alpha p}$ tubes $R_{\gamma}$, and when there is such a point. Note that the result is trivial if $\lambda>1$, so we will assume $\lambda \leq 1$.

In the first case, we have the following estimate:

$$
\begin{aligned}
|E| & \geq \frac{\sum_{\gamma} \int_{E} \chi_{R_{\gamma}}}{\mu} \\
& \approx \sum_{\gamma} \frac{\left|R_{\gamma} \cap E\right|}{\left|R_{\gamma}\right|} \cdot \frac{\delta^{d}}{\mu} \\
& \gtrsim \frac{N \lambda \delta^{d}}{\mu} \\
& =N \delta^{2 d-p+\alpha p} \lambda^{p}\left(\frac{\lambda^{1-p} \delta^{p-d-\alpha p}}{\mu}\right) \\
& =N \delta^{2 d-p+\alpha p} \lambda^{p} .
\end{aligned}
$$

Note that for $\lambda<\delta$, we can verify (3.16) using $\mu=C \delta^{-(d-1)}$. Due to the fact that the tubes must be in the direction of light rays, $C \delta^{-(d-1)}$ is the most tubes which could possibly intersect at a point (with $C$ depending only on $d$ ). So from now on we assume that $\delta \leq \lambda \leq 1$.

For the second case, let $x_{0}$ be a point contained in at least $\mu$ tubes. We consider the tubes $\widetilde{R}_{\gamma}$ which contain $x_{0}$. Let $B\left(x_{0}, \frac{\lambda}{C_{0}}\right)$ be the ball around $x_{0}$ of radius $\frac{\lambda}{C_{0}}$. Then for $C_{0}$ sufficiently large,

$$
\left|\left(\widetilde{R}_{\gamma} \cap E\right) \backslash B\left(x_{0}, \frac{\lambda}{C_{0}}\right)\right| \gtrsim \lambda \delta^{d} .
$$

Observe that $\left(\widetilde{R}_{\gamma} \cap E\right) \backslash B\left(x_{0}, \frac{\lambda}{C_{0}}\right)$ and $\left(\widetilde{R}_{\gamma^{\prime}} \cap E\right) \backslash B\left(x_{0}, \frac{\lambda}{C_{0}}\right)$ are disjoint if the angle between $\widetilde{R}_{\gamma}$ and $\widetilde{R}_{\gamma^{\prime}}$ is $\gtrsim \frac{\delta}{\lambda}$. There at most $\lambda^{1-d}$ tubes containing $x_{0}$ which are 
within an angle $\frac{\delta}{\lambda}$ of $\widetilde{R}_{\gamma}$, so we have the following:

$$
\begin{aligned}
|E| & \gtrsim \frac{\mu}{\lambda^{1-d}}\left(\lambda \delta^{d}\right) \\
& =\mu \lambda^{d} \delta^{d} \\
& =N \delta^{2 d-p+\alpha p} \lambda^{p}\left(\frac{\mu}{N \lambda^{p-d} \delta d-p+\alpha p}\right), \\
\frac{\mu}{N \lambda^{p-d} \delta d-p+\alpha p} & =\frac{1}{N} \lambda^{-2 p+d+1} \delta^{2 p-2 d-2 p \alpha} \\
& \gtrsim \lambda^{-2 p+d+1} \delta^{2 p-d-2 p \alpha} .
\end{aligned}
$$

In (3.25) we used the fact that $N \lesssim \delta^{-d}$. We are done once we show

$$
\lambda^{-2 p+d+1} \delta^{2 p-d-2 p \alpha} \gtrsim 1 .
$$

For $p \leq \frac{d+1}{2}, \alpha=\frac{1}{2 p}$,

$$
\begin{aligned}
\lambda^{-2 p+d+1} \delta^{2 p-d-2 p \alpha} & \geq \delta^{-2 p+d+1} \delta^{2 p-d-1} \\
& =1 .
\end{aligned}
$$

For $p \geq \frac{d+1}{2}, \alpha=1-\frac{d}{2 p}$,

$$
\begin{aligned}
\lambda^{-2 p+d+1} \delta^{2 p-d-2 p \alpha} & \geq 1^{-2 p+d+1} \delta^{2 p-d-(2 p-d)} \\
& =1 .
\end{aligned}
$$

\section{REFERENCES}

1. J. Bourgain, Besicovitch type maximal operators and applications to Fourier analysis, Geom. Funct. Anal. 1 (1991), no. 2, 147-187. MR1097257 (92g:42010)

2. L Estimates for cone multipliers, Geometric Aspects of Functional Analysis (Israel, 1992-1994, Oper. Theory Adv. Appl., no. 77, Birkhäuser Basel, 1995, pp. 41-60. MR.1353448 (96m:42022)

3. L. Carleson and P. Sjolin, Oscillatory integrals and a multiplier problem for the disc, Studia Math. 44 (1972), 287-299. MR0361607 (50:4052)

4. A. Córdoba, A note on Bochner-Riesz operators, Duke Math. J. 46 (1979), no. 3, 505-511. MR0544242 (80m:42025)

5. Some remarks on the Littlewood-Paley theory, Rend. Circ. Mat. Palermo (1981), 75-80, Suppl. 1. MR0639467 (83i:42015)

6. C. Fefferman, The multiplier problem for the ball, Annals of Math. 94 (1971), 330-336. MR $0296602(45: 5661)$

7. $\longrightarrow$ A note on spherical summation multipliers, Israel J. Math. 15 (1973), 44-52. MR0320624 (47:9160)

8. M. Jodeit, A note on Fourier multipliers, Proc. Amer. Math. Soc. 27 (1971), 423-424. MR 0270072 (42:4965)

9. I. Łaba and T. Wolff, A local smoothing estimate in higher dimensions, J. d'Analyse Math. 88 (2002), 149-171. MR1956533 (2005b:35015)

10. S. Lee, Improved bounds for Bochner-Riesz and maximal Bochner-Riesz operators, Duke Math. J. 122 (2004), no. 1, 205-232. MR2046812 (2005e:42042)

11. H. Luers, On Riesz means with respect to a cylindric distance function, Analysis Mathematica 14 (1988), 175-184. MR0981434 (90c:42020)

12. G. Mockenhaupt, A note on the cone multiplier, Proc. Amer. Math. Soc. 117 (1993), no. 1, 145-152. MR.1098404 (93c:42015)

13. G. Mockenhaupt, A. Seeger, and C. D. Sogge, Wave front sets, local smoothing and Bourgain's circular maximal theorem, Annals of Math. 136 (1992), 207-218. MR1173929 (93i:42009) 


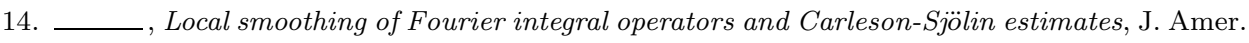
Math. Soc. 6 (1993), no. 1, 65-130. MR.1168960 (93h:58150)

15. A. Seeger, Some inequalities for singular convolution operators in $L^{p}$-spaces, Trans. Amer. Math. Soc. 308 (1988), no. 1, 259-272. MR0955772 (89j:42015)

16. Endpoint inequalities for Bochner-Riesz multipliers in the plane, Pacific J. Math. 174 (1996), no. 2, 543-553. MR1405600 (97j:42005)

17. J. Skarabot, Bounds for the Besicovitch type maximal operator, Ph.D. thesis, University of Wisconsin-Madison, 1997.

18. E. Stein, Harmonic analysis, Princeton University Press, 1993. MR.1232192 (95c:42002)

19. T. Tao, Sharp bilinear restriction estimates for elliptic surfaces, Geom. Funct. Anal. 13 (2003), no. 6, 1359-1384. MR2033842 (2004m:47111)

20. T. Tao, A. Vargas, and L. Vega, A bilinear approach to the restriction and Kakeya conjectures, J. Amer. Math. Soc. 11 (1998), 967-1000. MR1625056 (99f:42026)

21. T. Wolff, Local smoothing type estimates on $L^{p}$ for large p, Geom. Funct. Anal. 10 (2000), 1237-1288. MR $1800068(2001 \mathrm{k}: 42030)$

Department of Mathematics, University of Wisconsin-Madison, 480 Lincoln Drive, MADISON, WisCONSIN 53706

Current address: Department of Mathematics, Shippensburg University, 1871 Old Main Drive, Shippensburg, Pennsylvania 17257-2299

E-mail address: pttaylor@ship.edu 